\title{
Metal abundances in the cool cores of galaxy clusters ${ }^{\star}$
}

\author{
S. De Grandi ${ }^{1}$ and S. Molendi ${ }^{2}$ \\ 1 INAF - Osservatorio Astronomico di Brera, via E. Bianchi 46, 23807 Merate (LC), Italy \\ e-mail: sabrina.degrandi@brera.inaf.it \\ 2 INAF - IASF Milano, via Bassini 15, 20133 Milano, Italy \\ e-mail: silvano@iasf-milano.inaf.it
}

Received 22 June 2009 / Accepted 27 August 2009

\begin{abstract}
We use XMM-Newton data to carry out a detailed study of the $\mathrm{Si}, \mathrm{Fe}$ and $\mathrm{Ni}$ abundances in the cool cores of a representative sample of 26 local clusters. We performed a careful evaluation of the systematic uncertainties related to the instruments, the plasma codes and the spectral modeling, finding that the major source of uncertainty is the plasma codes. Our $\mathrm{Si}, \mathrm{Fe}, \mathrm{Ni}, \mathrm{Si} / \mathrm{Fe}$ and $\mathrm{Ni} / \mathrm{Fe}$ distributions feature only moderate spreads (from $20 \%$ to $30 \%$ ) around their mean values strongly suggesting similar enrichment processes at work in all our cluster cores. Our sample-averaged $\mathrm{Si} / \mathrm{Fe}$ ratio is comparable to those measured in samples of groups and high luminosity ellipticals, implying that the enrichment process in ellipticals, dominant galaxies in groups and BCGs in clusters is quite similar. Although our $\mathrm{Si} / \mathrm{Fe}$ and $\mathrm{Ni} / \mathrm{Fe}$ abundance ratios are fairly well constrained, the large uncertainties in the supernova yields prevent us from making a firm assessment of the relative contribution of type Ia and core-collapsed supernovae to the enrichment process. All that can be said with some certainty is that both contribute to the enrichment of cluster cores.
\end{abstract}

Key words. X-rays: galaxies: clusters - galaxies: clusters: general - supernovae: general - galaxies: abundances - cooling flows intergalactic medium

\section{Introduction}

The intra-cluster medium (ICM) that fills the deep potential well of galaxy clusters is rich in metals. In the high mass regime of rich clusters $\left(M_{\mathrm{vir}} \gtrsim 10^{14} M_{\odot}\right)$, where temperatures and densities of the gas are of the order of $3-10 \mathrm{keV}$ and $10^{-3} \mathrm{~cm}^{-3}$, respectively, the ICM is heavily ionized and the main processes producing emission lines are collisions of ions with electrons in a plasma in collisional ionization equilibrium. Since the elements are well confined within the cluster potential well, they accumulate over the whole cluster history and retain important information on cluster formation and evolution. For gas temperatures $\gtrsim 3 \mathrm{keV}$ the most prominent emission lines are from Helike and H-like K-shell transitions of $\mathrm{Fe}($ at $\sim 7 \mathrm{keV})$, along with less pronounced $\mathrm{K}$-shell lines of $\mathrm{S}, \mathrm{Si}$ (at $\sim 2 \mathrm{keV}$ ), $\mathrm{Ar}, \mathrm{Ca}$ and $\mathrm{Ni}($ at $\sim 8 \mathrm{keV})$. Temperature and abundances of the ICM are measured at the same time from the X-ray spectrum: the temperature is measured from the continuum emission that is almost entirely given by thermal bremsstrahlung, whereas the abundance of an element is derived by measuring the equivalent width of the line (once the continuum is known), that is directly proportional to the ion-to-hydrogen concentration ratio (see Kaastra et al. 2008 , for a review of the thermal radiation processes in clusters). While in principle the abundance measurement is straightforward, in practice various sources of uncertainties are present, amongst which (1) the accuracy of the atomic physics; (2) the moderate spectral resolution of the current imaging instruments which often results in line blending; this is particularly severe for L-shell blends where the transitions are not entirely understood; (3) the presence of temperature gradients in the ICM, especially

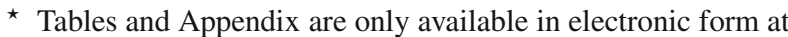
http://www . aanda.org in the cluster cores, that need specific spectral modeling. With the advent of the Chandra and XMM-Newton satellite, carrying detectors with both high spatial and spectral capabilities, the statistical quality of cluster spectra (in particular for cool core regions) improved dramatically. While on the one hand the statistical errors associated with the abundance measurements have greatly decreased, on the other hand surprisingly little attention has been devoted to systematic sources of uncertainties which, under these circumstances, are likely to play an important role.

In this paper, our first goal is to provide a robust measurement of the distribution of abundances and abundance ratios of the chemical elements in the cores of a representative sample of nearby and bright cool core clusters. In this context, "robust estimate" essentially means that we will include in the error budget a careful evaluation of the systematic uncertainties potentially affecting our data. We will consider both instrument related systematics and plasma code systematics. We stress that in this paper we are interested in the measure of the global abundances in the central region of each cluster, not in the analysis of radial profiles. Moreover, these central regions will provide us with the maximum photon statistics because of their very intense central surface brightness peaks, thereby allowing us to explore the most subtle sources of systematic errors and reliably measure the most abundant elements observable in the ICM (e.g. Si, Fe and Ni).

The elements observed in the ICM are produced through thermo-nuclear nucleo-synthesis in supernova (SN) explosions occurring in the member early-type galaxies (Arnaud et al. 1992; Renzini et al. 1993), and they are eventually ejected/diffused into the ICM through galactic winds (for a review see Bland-Hawthorn et al. 2007) and ram pressure stripping (e.g. Schindler \& Diaferio 2008, and references therein). Supernovae are classified on the basis of their progenitor models. Two main groups of SNe are known: type Ia supernovae (SNIa) that are 
derived from an accreting white dwarf in a binary system, and core-collapsed supernovae ( $\mathrm{SNcc}$ ) whose progenitors are single massive stars ( $\left.\gtrsim 8 M_{\odot}\right)$. One of the most important quantities that theoretical models of SNe provide are the yields of the various chemical elements, namely the mass per element and per SN event. While SNIa produce mostly $\mathrm{Fe}, \mathrm{Ni}$ and Si-group metals (i.e. $\mathrm{Si}, \mathrm{S}, \mathrm{Ar}, \mathrm{Ca}$ ), the $\mathrm{SNcc}$ ejecta are rich in $\alpha$-elements (i.e. $\mathrm{O}$, $\mathrm{Ne}, \mathrm{Mg}$, as well as few Si-group elements). In the $90 \mathrm{~s}$ BeppoSAX and $A S C A$ observations revealed that the centers of cool core clusters always display an excess (with respect to the external cluster regions) of $\mathrm{Fe}$ around the central cluster galaxy (e.g. Fukazawa et al. 2000; De Grandi \& Molendi 2001; Leccardi \& Molendi 2008a). The iron mass inferred from this excess is consistent with it being entirely produced by the giant galaxy itself (e.g. De Grandi et al. 2004; Böhringer et al. 2004), which is always found in these systems. Other elements such as $\mathrm{Mg}$, Si and $\mathrm{S}$ show abundance peaks in cluster cores (e.g. Fukazawa et al. 1998; Finoguenov et al. 2000, 2001; Tamura et al. 2004; Sato et al. 2009a). Under the assumption that the sole sources of metals are the two types of SNe, observations of the $\alpha$-elements/Fe abundance ratios coupled with the knowledge of the SNe chemical yields allow the determination of the SNIa and SNcc proportion within each cluster. Finoguenov et al. (2000) found an increasing $\mathrm{Si} / \mathrm{Fe}$ ratio with radius in clusters, indicating a greater predominance of SNcc enrichment at large radii, while the innermost parts appeared dominated by SNIa products. However, work based on data taken with the more recent XMM-Newton and SUZAKU observatories have not fully confirmed this overall picture for rich clusters. Tamura et al. (2004), studying the spatial distributions of metals in a sample of cool core clusters observed with XMM-Newton, found uniform $\mathrm{Si} / \mathrm{Fe}$ and $\mathrm{S} / \mathrm{Fe}$ ratios within the ICM, but increasing $\mathrm{O} / \mathrm{Fe}$ (although this ratio was prone to large uncertainties). SUZAKU observations confirm these findings (e.g. Sato et al. 2007a; Matsushita \& Suzaku SWG team 2008). The relative proportion of SNIa and SNcc found by XMM-Newton and SUZAKU observations are in rough agreement (Sato et al. 2007a), although these results were achieved using of specific collections of SNe yields (e.g. Iwamoto et al. 1999; Nomoto et al. 2006). None of the combinations of the theoretical SNIa and SNcc yields available in the literature have reproduced the overall elemental pattern in cluster cores (fits were not formally acceptable based on the $\chi^{2}$ values; e.g. Baumgartner et al. 2005; de Plaa et al. 2007; Sato et al. 2007a). There are at least two possible solutions to this problem: the existence of an additional source of elements other than SNIa and SNcc (see Finoguenov et al. 2002; Baumgartner et al. 2005; Mannucci et al. 2006), or, the need for a revision of the theoretical SNe models (e.g. Young \& Fryer 2007). Interestingly, a systematic exploration of the full range of uncertainties of theoretical yields was last provided by Gibson et al. (1997), although since then, several new compilations of SNcc yields have become available (e.g. Chieffi \& Limongi 2004; Nomoto et al. 2006) along with new yields for SNIa (Iwamoto et al. 1999). As pointed out by Gibson et al. (1997), the relative contribution of SNIa and SNcc to the Fe abundance in the ICM depends crucially upon the adopted theoretical SNe models.

The second main goal of this paper is to provide a critical assessment of the relative contributions of the SNIa versus SNcc to the enrichment process. Contrary to other similar works present in the recent literature (e.g. de Plaa et al. 2007), we do not neglect the uncertainties associated with the current theoretical yields and explore how they affect the derived SNIa fraction.

This paper is organized as follows. In Sect. 2 we present the sample, in Sect. 3 we describe the cleaning process of the raw
XMM-Newton archival data, and, in Sect. 4, we concentrate on the spectral analysis and on the choice of the best fitting model. In Sect. 4 we also discuss the systematic uncertainties related to imperfections in the cross-calibration between the three EPIC instruments. In Sect. 5 we present results on the derived distributions of metal abundances and abundance ratios. In this same section we compare results from two different choices for the spectral codes. In Sect. 6 we discuss the relative contribution to the overall enrichment process of different SNe types. In Sect. 7 we discuss our findings and in Sect. 8 we summarize our main results.

We assume $H_{0}=70 \mathrm{~km} \mathrm{~s}^{-1} \mathrm{Mpc}^{-1}$ and $\Omega_{\Lambda}=0.7$; the source redshifts are all extracted from the NASA/IPAC Extragalactic Database (NED). All metallicity measurements we show in this paper are relative to the photospheric solar abundances of Anders \& Grevesse (1989). In this set of abundances, Fe has a number density of $4.68 \times 10^{-5}$, Si $3.55 \times 10^{-5}$ and Ni $1.78 \times 10^{-6}$, all number densities are relative to $\mathrm{H}$. All uncertainties shown are $1 \sigma$ confidence level (i.e. $68 \%$ ).

\section{The sample}

Starting from the flux-limited sample of 55 galaxy clusters listed by Edge et al. (1990) and the ROSAT PSPC analysis of this sample performed by Peres et al. (1998), we have selected all the objects with a mass deposition rate different from zero (see Table 5 in Peres et al. 1998). Among these clusters we have chosen all those that were observed with XMM-Newton EPIC within April 2008, with the exclusion of clusters whose observations are strongly affected by soft protons (e.g. Abell 644 and Abell 2142). The 32 clusters that meet these requirements are listed in Table 1 (available online only).

\section{Data preparation}

We reprocessed the Observation Data Files (ODF) using the Science Analysis System (SAS) version 7.0.0. After the production of the calibrated event lists for the EPIC MOS1, MOS2 and pn observations with emchain and epchain tasks, we performed a soft proton cleaning using a double filtering process.

We first removed soft protons spikes by screening the light curves produced in 100 bins in the $10-12 \mathrm{keV}$ band and applying an appropriate threshold for each instrument, generally a threshold of $0.20 \mathrm{cts} \mathrm{s}^{-1}$ for MOS1 and MOS2, and of $0.60 \mathrm{cts} \mathrm{s}^{-1}$ for $p n$. Then to eliminate possible residual flares contributing below $10 \mathrm{keV}$, we extracted a light curve in the $2-5 \mathrm{keV}$ band and fitted the histogram produced from this curve with a Gaussian distribution. To generate the final filtered event file we rejected all events registered at times when the count rate was more than $3 \sigma$ from the mean of this distribution. Finally, we filtered event files according to FLAG $(\mathrm{FLAG}==0)$ and PATTERN (PATTERN $\leq 12$ for MOS and PATTERS $==0$ for $p n$ ) criteria. The resulting effective exposure times of the observations are reported in Table 1 (available online only).

Using the cleaned event files we extracted spectra from a circular region centered on the cluster emission peaks. The physical radius of this region is $0.5 r_{\text {cool }}$, where $r_{\text {cool }}$ is the cooling radius computed by Peres et al. (1998). With this choice of the extraction radius, we sample a significative portion of the cool core and we assure that the extracted region is contained within the EPIC field of view for all our clusters. Prominent point-like sources have been removed from the extraction region. Other authors (e.g. de Plaa et al. 2007; Rasmussen \& Ponman 2007) 
select their extraction regions as fixed fractions of the cluster scaling radius, e.g. de Plaa et al. (2007) used 0.2 of $r_{500}$, where $r_{500}$ is the radius encompassing a spherical density contrast of 500 with respect to the critical density. By converting our extraction radii in $r_{500}$ units we find a rather peaked distribution centered around $0.08 r_{500}$.

\section{Spectral analysis}

In this paper we wish to measure abundances for $\mathrm{Si}, \mathrm{Fe}$ and $\mathrm{Ni}$. To achieve robust estimates, we choose to make our measures from the K-shell lines only, avoiding L-shell blends where uncertainties associated with the atomic physics are larger. We achieve this by setting a lower energy threshold of $1.8 \mathrm{keV}$ which includes Si K-shell emission (2 keV) and excludes the Fe and $\mathrm{Ni}$ L-shell blends ( 1. $-1.5 \mathrm{keV})$.

The background subtraction for the high surface brightness core regions of cool core clusters is less critical than in the case of more external low surface brightness regions. We therefore decided to subtract the background using blank-sky fields instead of proceeding with a more detailed modeling of the different background components. The blank-sky fields for EPIC MOS and $p n$ were produced by Leccardi \& Molendi (2008a) (see Appendix B "The analysis of blank field observations" in their paper) by analysing a large number of observations for a total exposure time of $\sim 700 \mathrm{ks}$ for MOS and $\sim 500 \mathrm{ks}$ for $p n$.

We refined our background analysis by also performing a background rescaling for each observation separately to account for temporal variations of the background. We estimated the background intensity from spectra extracted from an external ring between $10^{\prime}$ and $12^{\prime}$ centered on the emission peak, taking into account only the $10-12 \mathrm{keV}$ band (to avoid possible extended cluster emission residuals in this region). We then rescaled the blank-sky fields background to the local value. This procedure is especially important when deriving the nickel abundance from its emission lines at $\sim 8 \mathrm{keV}$. Indeed, in this hard spectral region, both the EPIC effective area and the surface brightness of the relatively low temperature cluster cores decrease rapidly, and the background becomes progressively more important.

All the spectral fits were performed with the XSPEC package (version 11.3.2, Arnaud 1996).

We analyzed each cluster spectrum with three different models: (1) a one temperature thermal model with the plasma in collisional ionization equilibrium (vmekal model in the XSPEC nomenclature), referred to as the $1 \mathrm{~T}$ model hereafter; (2) a two temperature thermal model (vmekal+vmekal), 2T model hereafter, and; (3) a multi-temperature model (vmekal+vmekal+vmekal+vmekal), 4T model hereafter.

All models were multiplied by the Galactic hydrogen column density, $N_{\mathrm{H}}$, determined by HI surveys (Kalberla et al. 2005) through the wabs absorption model in XSPEC. In the following sections we report results from spectral fits with $N_{\mathrm{H}}$ fixed at the weighted Galactic value only. We have also allowed $N_{\mathrm{H}}$ to vary in all three models finding no significant differences in the derived silicon, iron and nickel abundance values.

The redshifts are taken from the NASA/IPAC Extragalactic Database and were always left as free parameters to account for small calibration differences between the two MOS and the $p n$ cameras. We have allowed the temperatures of $1 \mathrm{~T}$ and $2 \mathrm{~T}$ models to vary freely, whereas in the 4T model temperatures of the four components were fixed at 1,2, 4 and $8 \mathrm{keV}$, respectively. The normalizations of the models and the elements $\mathrm{Si}, \mathrm{S}, \mathrm{Fe}$ and
Ni were left free to vary. Ar and $\mathrm{C}$ were joined to $\mathrm{S}$ as these elements are less abundant and we do not plan to study them in the course of this work.

As pointed out by Leccardi \& Molendi (2008a), when fitting spectra with XSPEC it is appropriate to allow the metallicities to assume negative values. This procedure is necessary to avoid underestimates on the derived metal abundances which could affect the measurements, especially for the case of low metallicity, statistically poor spectra (for a more detailed discussion of this point see Appendix A in Leccardi \& Molendi 2008a).

Abundances are measured relative to the solar photospheric values of Anders \& Grevesse (1989), where $\mathrm{Fe}=4.68 \times 10^{-5}$, $\mathrm{Si}=3.55 \times 10^{-5}$ and $\mathrm{Ni}=1.78 \times 10^{-6}$ (by number relative to $\mathrm{H}$ ). We have chosen these values to allow direct comparison with other works present in the literature. Spectra from all three EPIC instruments (MOS1, MOS2 and $p n$ ) were fitted individually.

In the course of our analysis, we found that spectra with less than about 3500 source counts could not be used to constrain the abundances of nickel and silicon, therefore we decided to eliminate from the sample all clusters having less than 3500 source counts in the adopted energy band (i.e. $1.8-10 \mathrm{keV}$ ) in at least one of the three EPIC instruments. The five clusters removed from the sample by adopting this criterion are: Abell 1644, Abell 1651, Abell 2065, Abell 2063 and Abell 3562.

We have also excluded Cygnus A because of its peculiar core emission. A powerful double-lobed radio galaxy, QSO B1957+405, featuring huge jets feeding very large hotspot regions which are well detected in X-rays resides in the core of this cluster. In the light of the difficulty of removing efficiently the emission associated with the radio galaxy from the spectra of the core region we preferred to exclude this cluster from the sample.

In the case of the nickel abundance measurement, we introduced a further selection criterion to exclude clusters that are background dominated in the hard part of the spectrum. We selected the 7-9 keV energy band redshifted at the cluster distance around the nickel line at $\sim 8 \mathrm{keV}$, and we have measured the relative difference between the source and background count rates, $\left(c r_{\text {sou }}-c r_{\text {bgk }}\right) / c r_{\text {bkg }}$, in that band. We then considered only nickel measurements from clusters with relative difference larger than 1 , namely clusters with source count rates which were at least twice the background count rate in the hard band. Applying this selection Virgo, Abell 262 and Abell 1060 are also excluded from the sample. Our $p n$ spectra are only mildly contaminated by the fluorescence line complex located around $8 \mathrm{keV}$ (e.g. Ness et al. 2009), the reason being that our spectra are extracted from the central regions of the $p n$ detector where, due to a hole in the pn electronics box, the fluorescence lines are weak.

In summary we have measured $\mathrm{Si}$ and $\mathrm{Fe}$ abundances from a sample of 26 clusters and $\mathrm{Ni}$ from a subsample of 23 systems.

\subsection{Addendum to the spectral analysis of the pn}

Molendi \& Gastaldello (2009), analyzing the $p n$ spectra of a long XMM-Newton observation of the Perseus cluster, first noted in the best-fit residuals the presence of a substantial structure around the Fe $\mathrm{K} \alpha$ line (see Sect. 3.1.1 and Fig. 2 in their paper). This structure is attributed to an incorrect modeling of the pn spectral resolution within the redistribution matrix.

Investigating this features extensively in the $p n$ spectra of our cluster sample, we found that the resolution mis-calibration can be compensated for by including a multiplicative component that performs a Gaussian smoothing of the spectral model (gsmooth in XSPEC). We set the width of the Gaussian kernel to be $4 \mathrm{eV}$ 

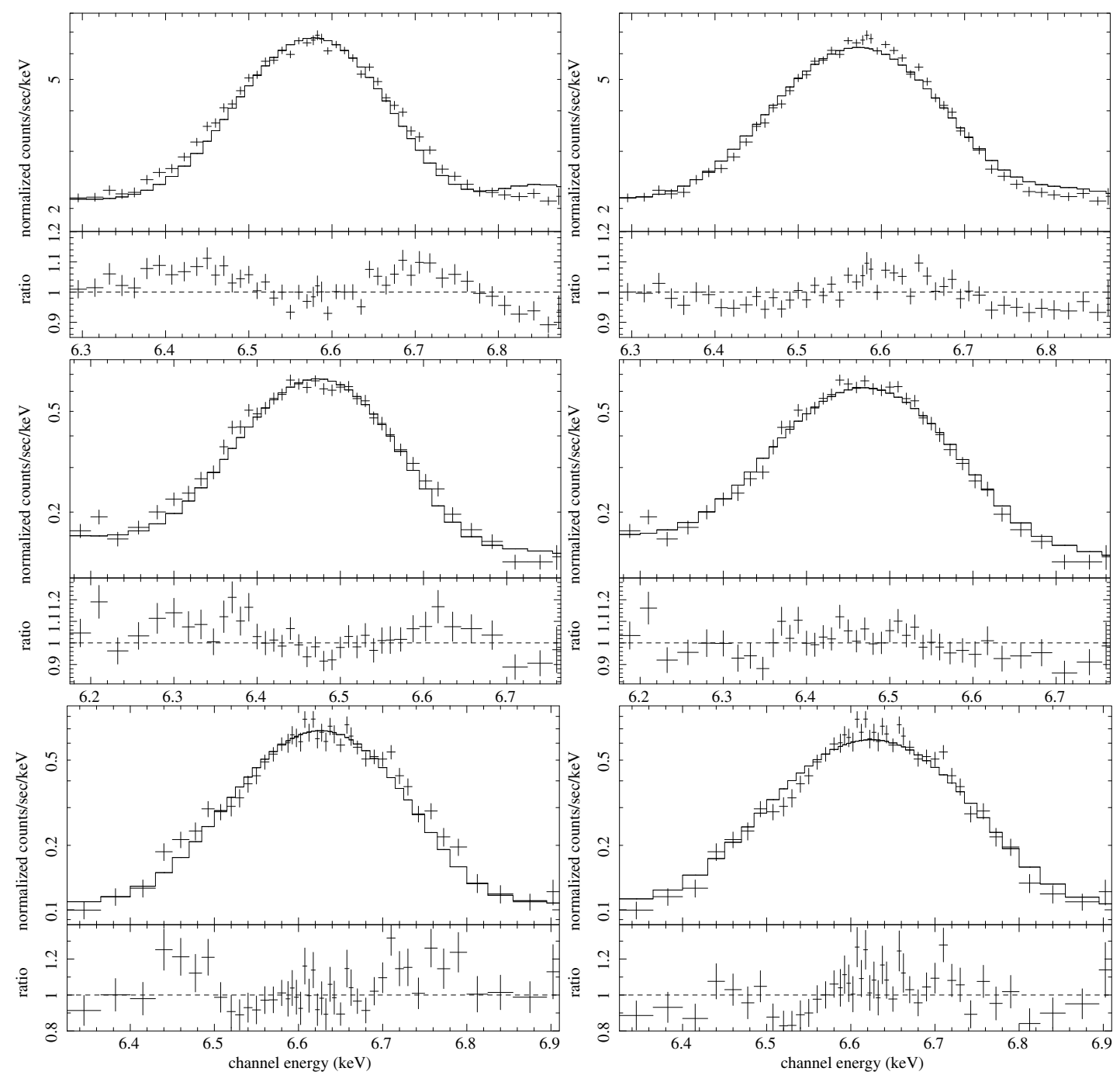

Fig. 1. EPIC-pn spectral data (crosses) and best-fit models (continuum lines) for 3 high counting statistics clusters: Perseus (on the top), 2A $0335+096$ (in the middle) and Centaurus (at the bottom). Panels on the left show results from a 4T model fit without Gaussian smoothing whereas plots on the right are smoothed. In all cases we also plot the data divided by the folded model. The spectra are rebinned for clarity. The energy range of each plot is $600 \mathrm{eV}$ centered on the $6.7 \mathrm{keV}$ line (redshifted at the cluster distance). In the panels on the left the "wings" around the Fe line are clearly visible in the residuals, an indication of the resolution mis-calibration of the redistribution matrix of the $p n$ (see discussion in Sect. 4.1).

$(F W H M)$ at $6 \mathrm{keV}$ and assume a power-law dependency of the width on the energy with an index of $\sim 1$.

Figure 1 shows the $p n$ spectra and best-fit residuals for three bright clusters: Perseus, 2A 0335+096 and Centaurus clusters. We have fitted the spectra with the $4 \mathrm{~T}$ model without and with the Gaussian smoothing. In the former cases (left panels in the figure), prominent residuals are present in the "wings" of the Fe line, while in the latter (right panels) the residuals are significantly reduced. In 13 (11) out of 26 cases the modified models applied to $p n$ spectra provide a substantially better fit (i.e. $\left.\Delta \chi^{2}>2.7(4.0)\right)$ than the unmodified models. We note that $\mathrm{Si}$ and Ni lines do not show similar residuals with respect to the best fit model, most likely because the statistical quality of the data is not as high ( $\mathrm{Si}$ and $\mathrm{Ni}$ ) and because the spectral resolution is significantly poorer $(\mathrm{Si})$.

After the application of the Gaussian smoothing, the crosscalibrations between the $p n$ and MOS improve. For instance, the relative differences, computed over the whole sample, between iron abundances estimated from $p n$ and MOS1 spectra, i.e. $\left(\mathrm{Fe}_{\mathrm{MOS} 1}-\mathrm{Fe}_{p n}\right) / \mathrm{Fe}_{p n}$, with the un-smoothed and smoothed $4 \mathrm{~T}$ model decrease from $12 \%$ to $7 \%$, whereas the ones between pn and MOS2 decrease from 9\% to $4 \%$ (errors on the given percentages are always $1 \%$ ).

\subsection{Choice of the best spectral model for each cluster}

In this section we discuss how we selected the model that best fits the spectral data among the 1T, 2T and 4T models described above.

Applying the statistical F-test between $1 \mathrm{~T}$ and $2 \mathrm{~T}$ (or $1 \mathrm{~T}$ and 4T) models, we find that only the first 6 out of 26 clusters with larger statistics (i.e. Perseus, Virgo, 2A $0335+96$, Centaurus, Abell 478 and Abell 4038) show overwhelming evidence of multi-temperature structure, namely an F-test probability, $P F$, smaller than $1 \%$ for all EPIC instruments.

The majority of the other clusters (17/26 for $2 \mathrm{~T}$ model and $22 / 26$ for $4 \mathrm{~T}$ model), however, show a significant improvement $(P F<1 \%)$ in at least one of the EPIC instruments. For these clusters, the relative differences between the metal abundances measured in each instrument with the $1 \mathrm{~T}$ and $2 \mathrm{~T}$ (or 1T and 4T) models are always smaller than $2-3 \sigma$ for $\mathrm{Fe}$ and $\mathrm{Si}$, and consistent with zero for $\mathrm{Ni}$. Therefore, from a purely statistical point of 
view, the choice of $1 \mathrm{~T}$ or multi-temperature (2T or $4 \mathrm{~T}$ ) models results in modest differences. Nevertheless, we point out that our spectra are extracted from the very central regions of cool core clusters and that these regions always display temperature gradients (e.g. Leccardi \& Molendi 2008b, and references therein). It follows that the best description of the spectrum of this plasma is through a multi-temperature model. For this reason and from the F-test results above, we decide to use a multi-temperature modeling for all the clusters in the sample.

An F-test between 2T and 4T models is not possible as the two models have the same numbers of degrees of freedom. However, relative differences between the metal abundances measured with the two models show that the systematic uncertainties associated with the different modeling of the data are below $2-3 \%$ for $\mathrm{Fe}$ and $\mathrm{Si}$ and consistent with zero for Ni. These systematic errors are of the same order as or smaller than the systematic errors on the abundances due to calibration differences between the three EPIC detectors (systematic errors given by the cross correlation between EPIC instruments will be discussed in Sect. 4.3). Therefore the two models are equivalent to describe our data. We choose to use the 4T model for all the clusters.

\subsection{EPIC cross-calibration issues}

For each cluster we compute the relative differences between iron, silicon, and nickel measured with MOS1, MOS2 and pn, and subsequently compute the weighted averages of these differences for the whole sample (i.e. 26 clusters for $\mathrm{Fe}$ and $\mathrm{Si}$, and 23 clusters for $\mathrm{Ni}$, results are from $4 \mathrm{~T}$ model). The resulting mean values for $\mathrm{Si}, \mathrm{Fe}$ and $\mathrm{Ni}$ are shown in Table 2 (available online only). From the results shown in this table it is clear that systematic errors are often dominant with respect to statistical ones for $\mathrm{Fe}$ and $\mathrm{Si}$, whereas $\mathrm{Ni}$ appears to be fully dominated by statistical uncertainties. Another important point is that the relative differences between MOS1 and MOS2 are smaller than the differences between MOS1 or MOS2 and $p n$.

To account for systematic differences between measures obtained from different detectors and spectral models we add in quadrature a $3 \%$ systematic error to each $\mathrm{Fe}$ and $\mathrm{Si}$ abundance measurement. The relative differences between iron, silicon, and nickel measured with MOS1, MOS2 and $p n$ computed over the whole sample, including the $3 \%$ systematic errors, are smaller than those computed without them and are always significant at less than $3 \sigma$ (these values are given in the second line relative to each element in Table 2).

For each cluster we compute an EPIC Fe and Si abundance by performing error weighted averages over the three detectors, with errors including the $3 \%$ systematic error described above. Errors on the EPIC Fe and Si abundances are computed by dividing the error weighted standard deviation of the EPIC abundance by the square root of 3 . To avoid errors on the EPIC abundances from falling below the systematic level, a $3 \%$ systematic error is summed in quadrature.

As shown in Table 2, nickel measurements are clearly dominated by statistical errors, nevertheless we decided for internal consistency to compute the nickel value for each cluster as described above for $\mathrm{Si}$ and $\mathrm{Fe}$. Indeed we expect $\mathrm{Ni}$ to be prone to the same systematics affecting $\mathrm{Si}$ and Fe.

The distribution of Fe and Si abundances is not very symmetric, which means that it is not particularly well represented by a Gaussian. We determined that the major cause is the presence of a few measurements with extremely small errors. Introducing a systematic error of $3 \%$ (see above) alleviates the problem considerably. The fact that the data is not normally distributed can be observed in Table 2: the Fe abundance measured with mekal, although the mean relative difference for MOS1 and MOS2 measures is small (2\%), and the mean relative difference for MOS1 and $p n$ is large, $(7 \%)$, the mean relative difference for MOS2 and $p n$ is modest, i.e. 2\%. Similar results are observed for $\mathrm{Si}$ measured with mekal and with Fe and Si measured with apec.

The final abundances are reported in Table 3 (available online only): Col. (1) is the cluster name, Cols. (2)-(4) are the EPIC error weighted averages of $\mathrm{Si}, \mathrm{Fe}$ and $\mathrm{Ni}$, respectively, with their $1 \sigma$ errors.

For completeness we have also computed all the abundances presented in Table 3 using the 2T model; as expected, we found only modest differences with respect to those estimated with the 4T model. The relative differences between the mean Fe, Si and $\mathrm{Ni}$ values computed for the whole sample with the $2 \mathrm{~T}$ and $4 \mathrm{~T}$ models are $-3 \% \pm 1 \%, 2 \% \pm 7 \%$ and $-1 \% \pm 9 \%$, respectively. In the case of Fe we detect a systematic error that is of the same order as the one associated with the choice of detector, while in the case of $\mathrm{Si}$ and $\mathrm{Ni}$ the indeterminations are sufficiently large to hide systematic errors of the same order.

\section{4. apec vs. mekal}

Mekal is not the only collisional ionization equilibrium (CIE) emission model in XSPEC. An alternative plasma code known as apec (Smith et al. 2001) is available. Some authors have performed comparisons between spectral fits performed with the two codes (e.g. Sanders \& Fabian 2006; de Plaa et al. 2007). Sanders \& Fabian (2006), analyzing a long Chandra observation of the core of Centaurus, found that while there are few differences between $\mathrm{Si}$ and $\mathrm{Fe}$ abundances estimated with the two codes, the Ni abundance found with apec is somewhat smaller than that found with mekal, a similar result, albeit with less statistical strength, was found by de Plaa and collaborators. Here we wish to investigate how the differences between the plasma codes impact on our abundance and abundance ratio measurements. To this end, we reanalyzed all our objects with a multitemperature model (vapec+vapec+vapec+vapec) analogous to the 4T mekal model which we call 4T apec. A comparison amongst apec metal abundances measured with the three EPIC instruments (see Table 2, available online only) provides results that are very similar to those found for mekal; including a systematic error of $3 \%$ on the measures alleviates the difference between the three instruments in much the same way (see Table 2) as it does for the 4T mekal fits. Mean metal abundances for Si, Fe and $\mathrm{Ni}$ and abundance ratios, $\mathrm{Si} / \mathrm{Fe}$ and $\mathrm{Ni} / \mathrm{Fe}$ obtained with apec and associated intrinsic scatters are reported in Table 4 (available online only). Comparing the sample average metal abundances measured with mekal with those obtained with apec we find that: $\mathrm{Fe}$ is almost unchanged, $\mathrm{Fe}_{\text {apec }} / \mathrm{Fe}_{\text {mekal }}=1.05 \pm 0.01$; $\mathrm{Si}$ is somewhat higher, $\mathrm{Si}_{\text {apec }} / \mathrm{Si}_{\text {mekal }}=1.11 \pm 0.02$ and $\mathrm{Ni}$ is lower, $\mathrm{Ni}_{\text {apec }} / \mathrm{Ni}_{\text {mekal }}=0.82 \pm 0.04$. The $\mathrm{Si} / \mathrm{Fe}$ ratio, as measured with apec, is slightly higher than that estimated with mekal, $(\mathrm{Si} / \mathrm{Fe})_{\text {apec }} /(\mathrm{Si} / \mathrm{Fe})_{\text {mekal }}=1.06 \pm 0.02$, while $\mathrm{Ni} / \mathrm{Fe}$ is substantially lower, $(\mathrm{Ni} / \mathrm{Fe})_{\text {apec }} /(\mathrm{Ni} / \mathrm{Fe})_{\text {mekal }}=0.77 \pm 0.04$.

In summary, while for $\mathrm{Fe}$ and $\mathrm{Si} / \mathrm{Fe}$ the systematic error associated with the cross-calibration between EPIC experiments, $3 \%$, are comparable to those related to the emission model, $5 \%$ and $6 \%$ respectively, for $\mathrm{Si}, \mathrm{Ni}$, and for $\mathrm{Ni} / \mathrm{Fe}$ the dominant source of error is the systematics related to the emission model, $11 \%, 18 \%$ and $23 \%$ respectively. The statistical errors on the mean $\mathrm{Si}, \mathrm{Fe}, \mathrm{Ni}, \mathrm{Si} / \mathrm{Fe}$ and $\mathrm{Ni} / \mathrm{Fe}$ are always dominated by systematic errors. 

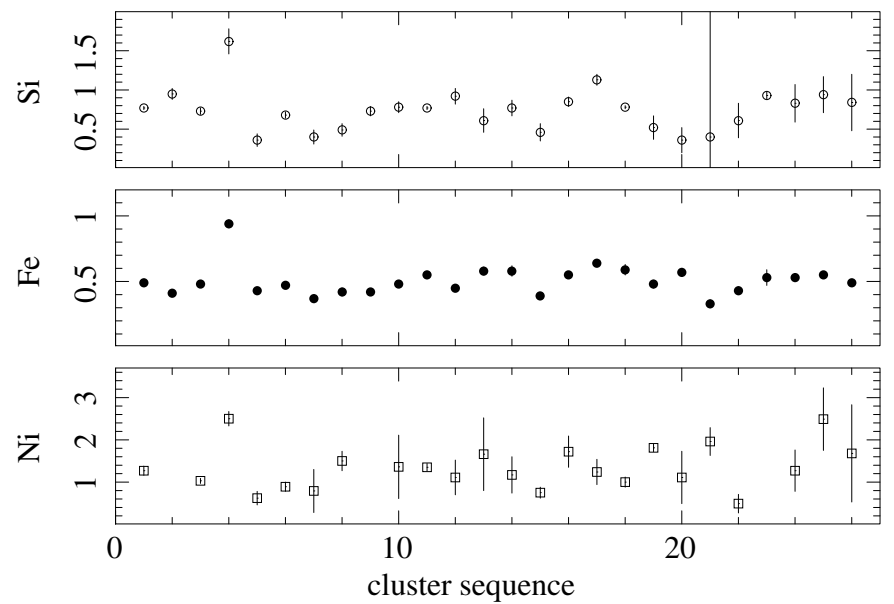

Fig. 2. Silicon (upper panel), iron (medium panel) and nickel (lower panel) abundances measured in the core region with $r<0.5 r_{\text {cool }}$ for the cluster sample. The total number of clusters is 26 for $\mathrm{Si}$ and $\mathrm{Fe}$, and 23 for Ni. Clusters are sorted by decreasing $p n$ counts statistics according to Table 3. Weighted mean values and intrinsic scatters of the relations are given in Table 4.

\section{Abundance results in cool cores}

\subsection{Mean iron, silicon and nickel abundances}

In Fig. 2 we show the abundances of silicon (upper panel), iron (middle panel) and nickel (lower panel) in the cool cores of our clusters (we use hereinafter results from the mekal model although results from both spectral codes are reported in Table 4, available online only). Visual inspection of the plots indicates that the abundance distribution show only small, $20 \%-30 \%$ variations with respect to the mean. The only object that is clearly out of the distributions is the Centaurus cluster whose core is uncommonly rich in metals (e.g. Sanders \& Fabian 2006).

To quantify the qualitative indications shown in Fig. 2 we have estimated the properties of the abundance distributions with a maximum likelihood algorithm that postulates a Gaussian parent distribution described by a mean and an intrinsic dispersion (Maccacaro et al. 1988). Our analysis shows that our abundance distributions feature a substantial intrinsic dispersion, roughly $30 \%$ for $\mathrm{Si}$ and $\mathrm{Ni}$ and $20 \%$ for $\mathrm{Fe}$. Best fitting means and intrinsic dispersions for all species are summarized in Table 4. To examine the influence of the outlier Centaurus cluster on the averages and scatters, we have repeated the computation excluding it from the sample, results are reported in Table 4. We do not find any dramatic change in the derived quantities, especially in the scatters of the abundance distribution that are only slightly smaller than those computed with the Centaurus cluster.

We have compared our $\mathrm{Fe}$ abundance estimates with those from Leccardi et al. (2009) who made measurements in a circular region with a radius of $0.05 r_{180}$, which roughly corresponds to our $1 / 2 r_{\text {cool }}$ extraction radius. We considered their cool cores systems excluding objects contained in our sample (these selections leads to a subsample of 13 clusters located in the $0.1-0.25$ redshift interval). Applying the maximum likelihood algorithm to their Fe distribution we find a mean Fe abundance of $0.49 \pm 0.02$ and a relative intrinsic scatter of $16 \% \pm 3 \%$, which are both in excellent agreement with our measurements.

The mean $\mathrm{Si}$ and $\mathrm{Fe}$ abundance values reported in Table 4 are also in good agreement with that found by Tamura et al. (2004) for the innermost regions of their cool core clusters with temperatures higher than $3 \mathrm{keV}$ (see Table 6 in Tamura et al. 2004).
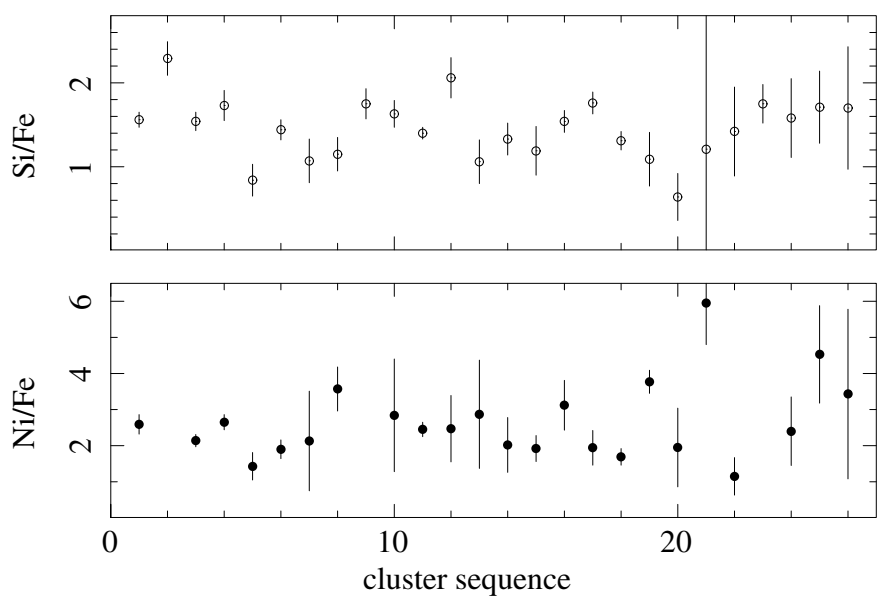

Fig. 3. Silicon-to-iron (upper panel) and nickel-to-iron (lower panel) ratios for the cluster sample.

On the contrary, de Plaa et al. (2007) found Si, Fe and $\mathrm{Ni}$ abundances somewhat lower than ours. By applying the maximum likelihood method to the abundances given in their Table A.1 (see de Plaa et al. 2007), we found an average Si of $0.39 \pm 0.02, \mathrm{Fe}$ of $0.35 \pm 0.02$ and $\mathrm{Ni}$ of $0.76 \pm 0.05$ (here and in the rest of this work abundances taken from the literature are rescaled to Anders \& Grevesse 1989), with intrinsic scatters for $\mathrm{Si}$ and $\mathrm{Fe}$ of $24 \%$ and $17 \%$, respectively. The lower abundance values are not surprising since the extraction regions used by de Plaa et al. (2007) are about three times larger than those considered in our work and because their sample contains both cool core and non cool core clusters.

Interestingly, the mean $\mathrm{Si}$ and $\mathrm{Fe}$ found by Rasmussen \& Ponman (2007) from a sample of galaxy groups are very similar to ours. From their Fig. 11 (panels b and c) we estimate a mean $\mathrm{Si}$ of $\sim 0.75$ and a mean $\mathrm{Fe}$ of $\sim 0.50$ within $0.08 \mathrm{r} / r_{500}$ which roughly corresponds to our $1 / 2 r_{\text {cool }}$ extraction radius.

\subsection{Silicon-to-iron and nickel-to-iron ratios}

In Fig. 3 we plot the silicon-to-iron (upper panel) and nickel-toiron (lower panel) ratio distributions for our objects. Using the maximum likelihood algorithm described in the previous section we computed the mean and the intrinsic dispersion for the $\mathrm{Si} / \mathrm{Fe}$ and $\mathrm{Ni} / \mathrm{Fe}$ distributions; results are shown in Table 4 (available online only). The relative scatter found for $\mathrm{Si} / \mathrm{Fe}$ is about $20 \%$, i.e. comparable to the one estimated for $\mathrm{Fe}$ and somewhat smaller than the one for $\mathrm{Si}$; for $\mathrm{Ni} / \mathrm{Fe}$ the scatter is only slightly greater $25 \%$. Interestingly, the Centaurus cluster, which features very high central abundances, is characterized by $\mathrm{Si} / \mathrm{Fe}$ and $\mathrm{Ni} / \mathrm{Fe}$ ratios very similar to those observed in other clusters.

As expected from the results on the $\mathrm{Si}$ and $\mathrm{Fe}$ abundances shown in the previous section, we find that our average $\mathrm{Si} / \mathrm{Fe}$ ratio is in good agreement with the value found by Tamura et al. (2004), $0.16 \pm 0.2$, in the innermost bin of their high temperature $(>3 \mathrm{keV})$ cool core clusters sample, whereas in de Plaa et al. (2007) the mean $\mathrm{Si} / \mathrm{Fe}$ and $\mathrm{Ni} / \mathrm{Fe}$ abundance ratios are $1.07 \pm 0.03$ and $2.07 \pm 0.13$, which are significantly lower than our values (the abundance ratios are computed with the maximum likelihood method applied to the data given in Table A.1 of de Plaa et al. 2007). As already mentioned, the observed difference between our abundance ratios and those from de Plaa et al. (2007) could derive from the different sample selection. 
We have compared our $\mathrm{Si} / \mathrm{Fe}$ ratio with those measured for a sample of groups (Rasmussen \& Ponman 2007) and of X-ray luminous elliptical galaxies (Humphrey \& Buote 2006). We note that in both papers abundances are reported in solar units which differ from ours, Grevesse \& Sauval (1998) for Rasmussen \& Ponman (2007) and Asplund et al. (2005) for Humphrey \& Buote (2006); to allow immediate comparison with our results the values shown below have been converted to Anders \& Grevesse (1989) units. The measure on the groups at scales comparable to those adopted for our clusters, $0.1 r_{500}$, (see Fig. $11 \mathrm{f}$ and Sect. 5.3 in Rasmussen \& Ponman 2007), provides a mean $\mathrm{Si} / \mathrm{Fe}$ ratio of 1.35 and a relative scatter of $32 \%$ which are both in broad agreement with ours. The measurement of the galaxies was made using data from high-luminosity ellipticals reported in Table 2 by Humphrey \& Buote (2006). We applied the maximum likelihood method used for our own data, deriving a mean $\mathrm{Si} / \mathrm{Fe}$ value of $1.50 \pm 0.07$ and a relative intrinsic scatter of $16 \% \pm 3 \%$, which are both in good agreement with our estimates for cool cores.

Summarizing, the average $\mathrm{Si} / \mathrm{Fe}$ ratio appears to be nearly constant from the galactic to the rich cluster scale suggesting a common enrichment scenario in these objects.

\section{SNla vs. SNcc}

There are indications that both type Ia and core-collapsed supernovae contribute significantly to the enrichment of the intergalactic medium in cores of cool core clusters (e.g. Ishimaru \& Arimoto 1997; Finoguenov et al. 2000; Gastaldello \& Molendi 2002). We try here to determine the relative proportion between the two SNe types using the silicon-to-iron and nickel-to iron abundance ratios normalized to the solar value.

The observed $\mathrm{X}_{i} / \mathrm{Fe}$ ratio, where $\mathrm{X}_{i}$ is the $i$ th element, can be expressed as a linear combination of the $\mathrm{X}_{i} / \mathrm{Fe}$ ratio expected from type Ia and core-collapsed supernovae (e.g. Gastaldello \& Molendi 2002):

$\left(\frac{\mathrm{X}_{i}}{\mathrm{Fe}}\right)_{\mathrm{obs}}=\xi \cdot\left(\frac{\mathrm{X}_{i}}{\mathrm{Fe}}\right)_{\mathrm{SNIa}}+(1-\xi) \cdot\left(\frac{\mathrm{X}_{i}}{\mathrm{Fe}}\right)_{\mathrm{SNcc}}$,

where

$\xi=\frac{M_{\mathrm{Fe}, \mathrm{SNIa}}}{M_{\mathrm{Fe}, \mathrm{SNIa}}+M_{\mathrm{Fe}, \mathrm{SNcc}}}$

is the SNIa iron-mass-fraction, i.e. the fraction of the iron mass synthesized in type Ia supernovae, $M_{\mathrm{Fe}, \mathrm{SNIa}}$ and $M_{\mathrm{Fe}, \mathrm{SNcc}}$ are respectively the total Fe mass ejected by SNIa and SNcc.

Matteucci \& Chiappini (2005) caution against an inappropriate use of Eq. (1) as in principle this equation is valid only if one is interested in the global $\mathrm{Fe}$ production (i.e., Fe in stars, galaxies and the ICM). Indeed, the model leading to Eq. (1) does not consider the finite timescale over which the gas is processed through stars and ignores the mechanism of chemical enrichment of the ICM from galaxies. However, as already noted by de Plaa et al. (2007) and Humphrey \& Buote (2006), Eq. (1) can be applied to the ICM, but the iron mass fractions that are derived from it should be interpreted as the iron mass fractions that would be needed to enrich the ICM, not as the actual iron mass fraction produced throughout the history of the cluster.

By inserting $\mathrm{Si} / \mathrm{Fe}$ and $\mathrm{Ni} / \mathrm{Fe}$ ratios expected for $\mathrm{SNIa}$ and $\mathrm{SNcc}$ and the mean observed $\mathrm{Si} / \mathrm{Fe}$ and $\mathrm{Ni} / \mathrm{Fe}$ ratios in Eq. (1) we estimate the SNIa iron-mass-fraction $\xi$. We compute $\xi$ by combining several theoretical SNe yields; the ones for SNIa originate from two physically different sets of models taken from
Iwamoto et al. (1999), namely the slow deflagration (W7 and W70) and the delayed detonation (WDDs and CDDs) explosion models. For core-collapsed supernovae we use the yields from Nomoto et al. (2006), Chieffi \& Limongi (2004) and Woosley $\&$ Weaver (1995); details on the models and the computation of the yields are provided in Appendix A (available online only). Since we have two equations, one for $\mathrm{Si} / \mathrm{Fe}$ the other for $\mathrm{Ni} / \mathrm{Fe}$, with one unknown, $\xi$, we are in a position to determine whether a given combination of Ia and core-collapsed models can adequately reproduce the observed ratios. One way of going about this (e.g. de Plaa et al. 2007) is to perform a $\chi^{2}$ fit using the observed $\mathrm{Si} / \mathrm{Fe}$ and $\mathrm{Ni} / \mathrm{Fe}$ ratios and associated errors as the data, the $\mathrm{Si}$ and Ni yields relative to Fe for SNIa and SNcc, namely $(\mathrm{Si} / \mathrm{Fe})_{\mathrm{SNIa}}$ and $(\mathrm{Si} / \mathrm{Fe})_{\mathrm{SNcc}}$, as constants and $\xi$ as a fitting parameter. We prefer to proceed differently, as has been noted that the uncertainties associated with the SNe yields are of the order of tens of percent (e.g., Woosley \& Weaver 1995; Gibson et al. 1997, P. Young priv. comm., and the scatter in the yields we have gathered from the literature fully confirms this, see Table A.1 and Appendix A, available online only), while our observed mean values of $\mathrm{Si} / \mathrm{Fe}$ and $\mathrm{Ni} / \mathrm{Fe}$ have errors smaller than $5 \%$, if we, for the time being, neglect the uncertainties associated with the spectral model. Consequently a robust estimate of $\xi$ should first take into account the uncertainties in the yields. Given the lack of precise information, we have assumed a uniform indetermination in the $\mathrm{Si}$ and $\mathrm{Ni}$ yields relative to $\mathrm{Fe}$ for SNIa and SNcc of 20\%. For each combination of SNIa and SNcc models we allow the $\mathrm{Si}$ and $\mathrm{Ni}$ yields relative to $\mathrm{Fe}$ for SNIa and SNcc to vary by $20 \%$ and, using the observed $\mathrm{Si} / \mathrm{Fe}$ and $\mathrm{Ni} / \mathrm{Fe}$ ratios, we determine the range of values of $\xi$ for which Eq. (1) is satisfied. For some combinations of SNIa and SNcc models, despite the generous $20 \%$ range, there are no values of $\xi$ satisfying Eq. (1), which implies that the observed $\mathrm{Si} / \mathrm{Fe}$ and $\mathrm{Ni} / \mathrm{Fe}$ cannot be reproduced for that particular combination of SNIa and SNcc models.

In Table 5 (available online only) we report ranges of values for $\xi$ for all possible combinations of Ia and core-collapsed supernovae models. The overall permitted range for $\xi$ is large $0.48-0.79$ and sensitive to the indetermination in the $\mathrm{SNe}$ yield ratios, indeed assuming $10 \%$ or $30 \%$ rather than $20 \%$ we derive a range of $0.55-0.73$ and $0.37-0.85$, respectively. Since the indetermination in the abundance ratios related to the emission model is comparable to the one in the yields we recompute the SNIa iron-mass-fraction assuming abundance ratios derived from apec rather than mekal. The results are similar, see Table A.1, the overall permitted range for $\xi$ is similar $0.49-0.90$, the major difference is in which of the combinations of SNIa and SNcc models provide valid solutions.

Equations (1) and (2) may be generalized to any atomic species and can therefore be used to obtain the $\mathrm{X}_{i}$ elements SNIa gas-mass-fraction. We have done so and derived the $\mathrm{Si}$ and $\mathrm{Ni}$ SNIa gas-mass-fraction ranges which are respectively $0.14-0.49$ and $0.26-0.88$.

By adopting abundance ratios derived from apec, we find broader ranges both for $\mathrm{Si}, 0.12-0.86$, and $\mathrm{Ni}, 0.01-0.90$. The reason for this difference rests in the smaller $\mathrm{Ni} / \mathrm{Fe}$ ratio found with apec. More specifically, the mekal value, 2.41, is relatively high when compared to the $\mathrm{Ni} / \mathrm{Fe}$ ratios predicted by $\mathrm{SNIa}$ and SNcc models. This implies that the observed Ni/Fe and Si/Fe ratios may only be reproduced by a limited combination of SNIa and SNcc models. Conversely, the apec value, 1.88, is closer to the mean $\mathrm{Ni} / \mathrm{Fe}$ ratio predicted by models and may be reproduced by a broader combination of SNIa and SNcc yields. 
The total Fe mass ejected by SNIa, $M_{\mathrm{Fe}, \mathrm{SNIa}}$, and by SNcc, $M_{\mathrm{Fe}, \mathrm{SNcc}}$ can be rewritten as:

$M_{\mathrm{Fe}, \mathrm{SNIa}}=N_{\mathrm{SNIa}} y_{\mathrm{Fe}, \mathrm{SNIa}}$

and

$M_{\mathrm{Fe}, \mathrm{SNcc}}=N_{\mathrm{SNcc}} y_{\mathrm{Fe}, \mathrm{SNcc}}$,

where $N_{\mathrm{SNIa}}$ and $N_{\mathrm{SNcc}}$ are respectively the total number of type Ia and core-collapsed supernovae and $y_{\mathrm{Fe}, \mathrm{SNIa}}$ and $y_{\mathrm{Fe}, \mathrm{SNcc}}$ are respectively the Fe yields per SNIa and SNcc.

We define $f \equiv N_{\mathrm{SNIa}} /\left(N_{\mathrm{SNIa}}+N_{\mathrm{SNcc}}\right)$ as the number ratio of supernovae type Ia over the total number of supernovae; combining Eq. (2) with Eqs. (3) and (4) we solve for $f$ :

$f=\frac{\xi y_{\mathrm{Fe}, \mathrm{SNcc}}}{\xi y_{\mathrm{Fe}, \mathrm{SNcc}}+(1-\xi) y_{\mathrm{Fe}, \mathrm{SNIa}}}$.

In Table 6 (available online only) we report estimates for $f$ obtained by inserting allowed ranges for $\xi$ in Eq. (5). We do not introduce any further indetermination in the Fe yields as the scatter in $\mathrm{Si}$ and $\mathrm{Ni}$ yields is much larger than the one in $\mathrm{Fe}$ and already accounted for through the scatter introduced in the $\mathrm{Si}$ and $\mathrm{Ni}$ yields relative to $\mathrm{Fe}$. As for $\xi$, the estimates are expressed in the form of permitted ranges. The overall permitted range for $f$ is rather large: $0.10-0.38$. Using abundance ratios estimated with the apec code we estimate a somewhat broader permitted range of $0.09-0.61$.

\section{Discussion}

In this work we have analyzed the central regions $\left(r<r_{\text {cool }} / 2\right)$ of a sample of 26 nearby and rich cool core clusters derived from the original B55 cluster sample (Edge et al. 1990). We have used these regions characterized by the highest cluster photon statistics to identify all the possible sources of systematic uncertainties in the abundance measurements of the most relevant elements observable in the X-ray spectral range between 1.8 and $10 \mathrm{keV}$, namely silicon, iron and nickel.

Our analysis shows that, within the cool cores of bright nearby clusters, metal abundances of $\mathrm{Si}, \mathrm{Fe}$ and in a few instances even Ni can be measured to a high precision. Indeed the precision is so high, particularly for $\mathrm{Fe}$, that we need to introduce a cross-calibration uncertainty of 3\% to reconcile measurements secured with different EPIC experiments. Thanks to the high statistical quality of our data, we find evidence for some intrinsic scatter in the element abundance with $\mathrm{Fe}$ around $20 \%$ and $\mathrm{Si}$ and $\mathrm{Ni}$ about $30 \%$ abundance ratios are also characterized by roughly the same intrinsic scatter.

The relatively modest scatter around mean values of $\mathrm{Si}, \mathrm{Fe}$ and $\mathrm{Ni}$ abundances and $\mathrm{Si} / \mathrm{Fe}$ and $\mathrm{Ni} / \mathrm{Fe}$ ratios indicates that, whatever the process responsible for the enrichment of the ICM, it works similarly in all objects. Even in the somewhat extraordinary case of the Centaurus cluster with its anomalously large metal content, the abundances relative to iron are perfectly compatible with those of the other clusters, indicating the presence of similar enrichment processes. Uncertainties in the abundance estimates associated with the specific choice of spectral model, i.e. $2 \mathrm{~T}$ rather than $4 \mathrm{~T}$ appear to be negligible (i.e., within the statistical uncertainties for $\mathrm{Si}$ and $\mathrm{Ni}$, and of the same order as the EPIC cross-calibration differences for iron).

The estimate of the abundances from our spectra may be thought of, with some simplification, as a two step process: in the first step the equivalent width of a given line is estimated; in the second step the equivalent width is converted into an abundance assuming the temperature derived by fitting the continuum. While the first step is relatively straight-forward and, at least for K-shell lines, leaves little room for ambiguity, the second, requiring estimates of collisional ionization and transition probabilities, is far more prone to differences. We have verified that the two plasma codes available within XSPEC provide somewhat different estimates for the metal abundances; the differences are not huge and are qualitatively similar to those found by other workers (Sanders \& Fabian 2006; de Plaa et al. 2007). However, given the high quality of our abundance estimates, they provide the dominant source of indetermination for $\mathrm{Si}(10 \%), \mathrm{Ni}(16 \%)$ and $\mathrm{Ni} / \mathrm{Fe}(22 \%)$ and contribute substantially in the case of $\mathrm{Fe}(4 \%)$ and $\mathrm{Si} / \mathrm{Fe}(\% 6)$.

We find that the $\mathrm{Si} / \mathrm{Fe}$ abundance ratio estimated for cool core clusters (this work; Tamura et al. 2004), shows a remarkably good agreement with the values found from samples of galaxy groups (Rasmussen \& Ponman 2007) and X-ray luminous elliptical galaxies (Humphrey \& Buote 2006). This similarity favors a common enrichment scenario for luminous elliptical galaxies and the cool cores of groups and clusters, i.e. a similar mix of SNIa and SNcc.

We have used our estimates of the $\mathrm{Si} / \mathrm{Fe}$ and $\mathrm{Ni} / \mathrm{Fe}$ abundance ratios to constrain the relative contribution of type Ia and core-collapsed supernovae to the enrichment process. To this end we have compiled a list of SNIa and SNcc yields from the literature. Simply by inspecting our yields (see Tables A.1 and A.2 available online only) it is rather obvious that the large differences, in the tens of \%, are bound to have a non-negligible impact on our estimates. The Fe normalized yields of $\mathrm{Si}$ and $\mathrm{Ni}$ both feature a scatter that is larger than the indetermination in the corresponding observed abundance ratios. Under the rather simplistic assumption of a $20 \%$ indetermination in the yields we find that, $f$, the fraction of Ia to Ia plus core-collapsed supernovae cannot be reconciled with 0 or 1 , in other words we need both Ia and core-collapsed supernovae to produce the observed ratios. This result appears to be rather solid; an indetermination of at least $50 \%$ is required to allow $f=0$ and then only for one specific combination of Ia and core-collapsed supernovae, an indetermination of more than $70 \%$ to have $f=1$.

Going beyond the qualitative statement that both SNIa and SNcc contribute to the enrichment of the ICM is quite hard. The accepted range for $f$, the ratio of type Ia $\mathrm{SN}$ to all $\mathrm{SNe}$, convolved over all the SNIa and SNcc combinations providing valid results, is rather large, from $10 \%$ to $40 \%$. Similarly we can say that roughly more than half $(48-79 \%)$ of the $\mathrm{Fe}$ is produced by SNIa; less than half of the $\mathrm{Si}(14-49 \%)$ is produced by SNIa and $\mathrm{Ni}$ is very poorly constrained (26-88\%). Increasing the indetermination on the yields beyond $20 \%$ will of course further enlarge the allowed ranges and result in even weaker constraints. We reiterate that the limiting factor is not the X-ray abundance estimates, as mean values can be constrained rather well even when allowing for systematics associated with instrument calibration and thermal emission model uncertainties, but rather is the large indetermination in the theoretical yields. A similar point was made some ten years ago by Gibson et al. (1997), a decade later the substantially improved observational constraints stand out in contrast with the lack of any similar advancement on the theoretical side.

Our analysis shows how difficult it is to determine the relative contribution of type Ia and core-collapsed supernovae to the enrichment of the ICM from global cool core measurements. An alternative approach is to measure radial profiles of metal abundances. In this case the variation of an abundance ratio such 
as $\mathrm{Si} / \mathrm{Fe}$, where the two elements are produced in different proportions by SNcc and SNIa, can be interpreted as evidence for a variation of the contribution of one type of $\mathrm{SN}$ with respect to the other. This kind of analysis has been attempted in the past, Finoguenov et al. (2000, 2001), using ASCA data, found that for most rich clusters in their sample the $\mathrm{Si} / \mathrm{Fe}$ rapidly increased when moving from small to large radii. Later work using XMM-Newton data did not confirm these findings; Tamura et al. (2004) find a flat $\mathrm{Si} / \mathrm{Fe}$ ratio for a sample of 19 clusters. Amongst the few objects in common between the ASCA and XMM-Newton samples are A3112 (Finoguenov et al. 2000) and A2052 (Finoguenov et al. 2001) for which ASCA data found $\mathrm{Si} / \mathrm{Fe}$ radial gradients and $X M M-N e w$ ton derived flat $\mathrm{Si} / \mathrm{Fe}$ profiles. Recent analysis of SUZAKU data (Sato et al. 2007b, 2009a,b; Komiyama et al. 2009) on a handful of groups and poor clusters leads to $\mathrm{Si} / \mathrm{Fe}$ profiles that are consistent with being constant out to at least $0.1 r_{180}$ and in some instances to $0.2 r_{180}$. Since at $0.1 r_{180}$ the average $\mathrm{Fe}$ abundance excess is about $1 / 2$ of what it is within $0.03 r_{180}$ (Leccardi \& Molendi 2008a), this seems to rule out the possibility that the transition from central excess to flat $\mathrm{Fe}$ profile might be associated with a change in $\mathrm{SN}$ type mix.

Future work on XMM-Newton, Chandra and SUZAKU data may change the situation somewhat by providing new abundance ratio measurements beyond $0.1 r_{180}$. However, given the considerable difficulties involved in estimating abundances at large radii (e.g. Leccardi \& Molendi 2008a), it is by no means clear if and by how much our estimates will improve. A major advancement will be provided by the first mission carrying a microcalorimeter, most likely the Japanese ASTRO-H. The ten fold increase in resolution at the $\mathrm{Si}$ line will allow us to reduce the background by an order of magnitude for the H-like Si line and by a factor of a few for the He-like Si triplet. This will translate into measures of $\mathrm{Si}$ abundances out to at least $0.3 r_{180}$ for a good number of nearby clusters.

\section{Summary}

We have performed a detailed study of the $\mathrm{Si}, \mathrm{Fe}$ and $\mathrm{Ni}$ abundances in the cores of 26 nearby cool core clusters. Our work may be divided into two main parts; the first relates to the measure of the abundances and the second to the estimate of the relative contribution of SNIa and SNcc to the ICM enrichment process. Regarding the first part, our main result may be summarized as follows.

- We find that systematic uncertainties associated with the different spectral modeling, namely a $2 \mathrm{~T}$ versus $4 \mathrm{~T}$ model, are below a few per cent $(2-3 \%)$.

- We find evidence for a $3 \%$ systematic error between Si and $\mathrm{Fe}$ abundance measures secured with the three EPIC detectors. The Ni abundances do not have the statistical quality to investigate such small systematic errors.

- We have verified that the mekal and apec plasma codes available in XSPEC give somewhat different abundances values. Given the high photon statistics of our spectra, they contribute significantly to the indetermination of $\mathrm{Fe}(4 \%)$ and $\mathrm{Si} / \mathrm{Fe}(6 \%)$ and are the dominant source of indetermination from $\mathrm{Si}(\sim 10 \%)$, Ni $(\sim 15 \%)$ and $\mathrm{Ni} / \mathrm{Fe}(\sim 20 \%)$.

- The final Si, Fe and Ni abundance distributions as well as the $\mathrm{Si} / \mathrm{Fe}$ and $\mathrm{Ni} / \mathrm{Fe}$ abundance ratio distributions of the sample show only moderate spreads (from $20 \%$ to $30 \%$ ) around their mean values (exact values are reported in Table 4).
These nearly constant distributions suggest similar ICM enrichment processes at work in all cluster cores.

- We find a remarkable uniformity in the observed $\mathrm{Si} / \mathrm{Fe}$ abundance ratio ranging from $\mathrm{X}$-ray luminous elliptical galaxies to the cool cores of groups and clusters. This tells us that, whatever the real proportion between different SNe types may be, the enrichment process of the hot gas associated with elliptical galaxies is likely the same in isolated ellipticals, dominant galaxies in groups and the brightest cluster galaxies in clusters.

Regarding the second part, we have used our estimates of the $\mathrm{Si} / \mathrm{Fe}$ and $\mathrm{Ni} / \mathrm{Fe}$ abundance ratios to constrain the relative contribution of SNIa and SNcc to the enrichment process of the ICM. We have considered a suite of 6 SNcc yields and 7 SNIa yields and, unlike previous studies, we have included both uncertainties associated with the observed abundance ratios and the theoretical yields for $\mathrm{Si}, \mathrm{Fe}$ and $\mathrm{Ni}$. Under the assumption that the indetermination on the yields currently available in the literature is of the order of $20 \%$, we find that:

- The SNIa iron mass fraction, $\xi$, overall permitted range is $0.48-0.79$.

- The SNIa silicon mass fraction and nickel mass fraction ranges are $0.14-0.49$ and $0.26-0.88$, respectively.

- The number ratio of SNIa over the total number of SNe, $f$, spans from 0.10 to 0.38 .

- The dominant source of uncertainty in the estimate of $\xi$ is errors on theoretical yields; conversely, the choice of spectral code has almost no impact.

Our analysis shows that the large uncertainties on the currently available yield tables prevent any precise estimate of the relative contribution of SNIa and SNcc, and that all that can really be said with some certainty is that they both contribute to the ICM enrichment process in the cool core regions. An alternative approach to estimate the relative contribution of SNIa and SNcc is to measure the difference between abundance ratios, such as $\mathrm{Si} / \mathrm{Fe}$, in the core and in the outer regions of individual systems. While early attempts have not provided definitive results, a more robust analysis of currently available data may furnish important constraints. The coming into operation of the first space-borne micro-calorimeter, most likely ASTRO-H, will undeniably allow a significant step forward.

Acknowledgements. We acknowledge useful discussions with Mariachiara Rossetti and Fabio Gastaldello. This research has made use of the NASA/IPAC Extragalactic Database (NED) which is operated by the Jet Propulsion Laboratory, California Institute of Technology, of the NASA's High Energy Astrophysics Science Archive Research Center (HEASARC), and, of the XMM-Newton archive.

\section{References}

Anders, E., \& Grevesse, N. 1989, Geochim. Cosmochim. Acta, 53, 197 Arimoto, N., \& Yoshii, Y. 1987, A\&A, 173, 23

Arnaud, K. A. 1996, in Astronomical Data Analysis Software and Systems V, ed. G. H. Jacoby, \& J. Barnes, ASP Conf. Ser., 101, 17

Arnaud, M., Rothenflug, R., Boulade, O., Vigroux, L., \& Vangioni-Flam, E. 1992, A\&A, 254, 49

Asplund, M., Grevesse, N., \& Sauval, A. J. 2005, in Cosmic Abundances as Records of Stellar Evolution and Nucleosynthesis, ed. T. G. Barnes, III, \& F. N. Bash, ASP Conf. Ser., 336, 25

Baumgartner, W. H., Loewenstein, M., Horner, D. J., \& Mushotzky, R. F. 2005, ApJ, 620, 680

Bland-Hawthorn, J., Veilleux, S., \& Cecil, G. 2007, Ap\&SS, 311, 87

Böhringer, H., Matsushita, K., Churazov, E., Finoguenov, A., \& Ikebe, Y. 2004, A\&A, 416, L21 
Chieffi, A., \& Limongi, M. 2004, ApJ, 608, 405

De Grandi, S., \& Molendi, S. 2001, ApJ, 551, 153

De Grandi, S., Ettori, S., Longhetti, M., \& Molendi, S. 2004, A\&A, 419, 7

de Plaa, J., Werner, N., Bleeker, J. A. M., et al. 2007, A\&A, 465, 345

Edge, A. C., Stewart, G. C., Fabian, A. C., \& Arnaud, K. A. 1990, MNRAS, 245, 559

Finoguenov, A., David, L. P., \& Ponman, T. J. 2000, ApJ, 544, 188

Finoguenov, A., Arnaud, M., \& David, L. P. 2001, ApJ, 555, 191

Finoguenov, A., Matsushita, K., Böhringer, H., Ikebe, Y., \& Arnaud, M. 2002, A\&A, 381, 21

Fukazawa, Y., Makishima, K., Tamura, T., et al. 1998, PASJ, 50, 187

Fukazawa, Y., Makishima, K., Tamura, T., et al. 2000, MNRAS, 313, 21

Gastaldello, F., \& Molendi, S. 2002, ApJ, 572, 160

Gibson, B. K., Loewenstein, M., \& Mushotzky, R. F. 1997, MNRAS, 290, 623

Grevesse, N., \& Sauval, A. J. 1998, Space Sci. Rev., 85, 161

Humphrey, P. J., \& Buote, D. A. 2006, ApJ, 639, 136

Ishimaru, Y., \& Arimoto, N. 1997, PASJ, 49, 1

Iwamoto, K., Brachwitz, F., Nomoto, K., et al. 1999, ApJS, 125, 439

Kaastra, J. S., Paerels, F. B. S., Durret, F., Schindler, S., \& Richter, P. 2008 Space Sci. Rev., 134, 155

Kalberla, P. M. W., Burton, W. B., Hartmann, D., et al. 2005, A\&A, 440, 775

Komiyama, M., Sato, K., Nagino, R., Ohashi, T., \& Matsushita, K. 2009, PASJ, 61,337

Leccardi, A., \& Molendi, S. 2008a, A\&A, 487, 461

Leccardi, A., \& Molendi, S. 2008b, A\&A, 486, 359

Leccardi, A., Rossetti, M. C., \& Molendi, S. 2009, A\&A, submitted

Lodders, K. 2003, ApJ, 591, 1220

Maccacaro, T., Gioia, I. M., Wolter, A., Zamorani, G., \& Stocke, J. T. 1988, ApJ 326,680
Mannucci, F., Della Valle, M., \& Panagia, N. 2006, MNRAS, 370, 773

Matsushita, K., \& Suzaku SWG team. 2008, Astron. Nachr., 329, 139

Matteucci, F., \& Chiappini, C. 2005, PASA, 22, 49

Molendi, S., \& Gastaldello, F. 2009, A\&A, 493, 13

Ness, J. U., Ehle, M., Gonzalez Riestra, R., et al. 2009, XMM-Newton Users' Handbook, http://xmm.esac.esa.int./external/xmm_user suport/documentation/uhb/index.html

Nomoto, K., Tominaga, N., Umeda, H., Kobayashi, C., \& Maeda, K. 2006, Nuclear Phys. A, 777, 424

Peres, C. B., Fabian, A. C., Edge, A. C., et al. 1998, MNRAS, 298, 416

Rasmussen, J., \& Ponman, T. J. 2007, MNRAS, 380, 1554

Renzini, A., Ciotti, L., D’Ercole, A., \& Pellegrini, S. 1993, ApJ, 419, 52

Salpeter, E. E. 1955, ApJ, 121, 161

Sanders, J. S., \& Fabian, A. C. 2006, MNRAS, 371, 1483

Sato, K., Tokoi, K., Matsushita, K., et al. 2007a, ApJ, 667, L41

Sato, K., Yamasaki, N. Y., Ishida, M., et al. 2007b, PASJ, 59, 299

Sato, K., Matsushita, K., \& Gastaldello, F. 2009a, PASJ, 61, 365

Sato, K., Matsushita, K., Ishisaki, Y., et al. 2009b, PASJ, 61, 353

Schindler, S., \& Diaferio, A. 2008, Space Sci. Rev., 134, 363

Scott, P., Asplund, M., Grevesse, N., \& Sauval, A. J. 2009, ApJ, 691, L119

Smith, R. K., Brickhouse, N. S., Liedahl, D. A., \& Raymond, J. C. 2001, ApJ, 556, L91

Tamura, T., Kaastra, J. S., den Herder, J. W. A., Bleeker, J. A. M., \& Peterson, J. R. 2004, A\&A, 420, 135

Werner, N., Durret, F., Ohashi, T., Schindler, S., \& Wiersma, R. P. C. 2008, Space Sci. Rev., 134, 337

Woosley, S. E., \& Weaver, T. A. 1995, ApJS, 101, 181

Young, P. A., \& Fryer, C. L. 2007, ApJ, 664, 1033 


\section{Appendix A:}

We have computed yields for $\mathrm{Si}, \mathrm{Fe}$ and $\mathrm{Ni}$ for different models of Ia and core-collapsed supernovae.

The ones for SNIa originate from two physically distinct sets of models taken from Iwamoto et al. (1999), namely the slow deflagration (W7 and W70) and the delayed detonation (WDDs and CDDs) explosion models. We have computed silicon, iron and nickel yields for a given SNIa model by summing over all stable isotopes the synthesized amount of $\mathrm{Si}, \mathrm{Fe}$ and Ni per SN event given in Table A.1 of Iwamoto et al. (1999).

For core-collapsed supernovae we use the yields from Nomoto et al. (2006), Chieffi \& Limongi (2004) and Woosley \& Weaver (1995). In these cases we first averaged the yields over the initial mass function (IMF) of the stellar population as follows:

$M_{i}=\frac{\int_{10 M_{\odot}}^{50 M_{\odot}} M_{i}(m) m^{-(1+x)} \mathrm{d} m}{\int_{10 M_{\odot}}^{50} m^{-(1+x)} \mathrm{d} m}$,

where $M_{i}(m)$ is the $i$ th element mass produced by a star of mass $m$. We consider two IMF: a standard Salpeter (1955) function with exponent $x=1.35$, and a top-heavy function with $x=0.95$ (Arimoto \& Yoshii 1987, thereafter AY), which predicts a relatively larger number of massive stars. The integration mass range is between 10 and 50 solar masses, in agreement with Nomoto et al. (2006).

The derived yields of $\mathrm{Si}, \mathrm{Fe}$ and $\mathrm{Ni}$ in units of solar masses per supernova explosion are reported in Table A.1. For corecollapsed supernovae we report results for the standard Salpeter and the AY top-heavy IMF.

Inspection of Table A.1 reveals how much yields differ from one model to the other. Fe is characterized by the smallest scatter, $10 \%$ for SNIa and $18 \%$ for SNcc, Si is less constrained, $27 \%$ for SNIa and $19 \%$ for SNcc, Ni features the largest variations, $46 \%$ for SNIa and $41 \%$ for SNcc. Note also that the choice of IMF produces only a modest impact on the yields.

The derived SNIa yields of $\mathrm{Si}$ and $\mathrm{Ni}$ relative to iron in Anders \& Grevesse (1989) solar units are reported in Table A.2. Ratios in Grevesse \& Sauval (1998) units can be easily computed by multiplying both the $\mathrm{Si} / \mathrm{Fe}$ and $\mathrm{Ni} / \mathrm{Fe}$ values in Table A.2 by 0.675 , whereas ratios in Lodders (2003) units are obtained by multiplying $\mathrm{Si} / \mathrm{Fe}$ by 0.65 and $\mathrm{Ni} / \mathrm{Fe}$ by 0.68 , and, ratios in Asplund et al. (2005) units by multiplying Si/Fe by 0.67 and $\mathrm{Ni} / \mathrm{Fe}$ by 0.72 (for the solar $\mathrm{Ni}$ we have used the updated value by Scott et al. 2009).

The derived yields of $\mathrm{Si}$ and Ni relative to iron in solar units for SNcc for different progenitor metallicities and IMFs are reported in Table A.2. Conversion factors for different solar units systems are the same as given above.

The yields relative to $\mathrm{Fe}$ for all our SNcc models are also listed in a recent review paper (Werner et al. 2008). Unfortunately some of the values reported in that paper are incorrect, in some cases, by a large amount, i.e. the Ni yields for the Woosley \& Weaver (1995) models. We have corresponded with the authors who are now considering publishing a revised version of their tables. 
S. De Grandi and S. Molendi: Metal abundances in the cool cores of galaxy clusters, Online Material p 2

Table A.1. Yields of elements in solar masses for different SNe models. Type Ia yields were calculated for each element for the W7, W70, WDD1, WDD2, WDD3 and CDD1, CDD2 models in Iwamoto et al. (1999). SN core-collapsed yields (including types Ib and Ic) were calculated from the results in Nomoto et al. (2006) integrating over a progenitor initial mass function. We have considered two different initial mass functions, the Salpeter function with index 1.35 and the AY top-heavy function with index 0.95 , and we have integrated between 10 and 50 solar masses. The initial metallicity of the progenitors is $Z=0.02$ (i.e. solar).

\begin{tabular}{lccccccc}
\hline \hline & \multicolumn{7}{c}{ SNIa } \\
\hline Element & W7 & W70 & WDD1 & WDD2 & WDD3 & CDD1 & CDD2 \\
\hline Si & 0.16 & 0.14 & 0.27 & 0.21 & 0.16 & 0.28 & 0.20 \\
$\mathrm{Fe}$ & 0.76 & 0.77 & 0.67 & 0.79 & 0.87 & 0.65 & 0.83 \\
$\mathrm{Ni}$ & 0.13 & 0.10 & 0.04 & 0.06 & 0.07 & 0.04 & 0.06 \\
\hline
\end{tabular}

\begin{tabular}{lcccccc}
\hline \hline & \multicolumn{7}{c}{ SNcc } \\
\hline Element & \multicolumn{7}{c}{ Nomoto etal. (2006) } & \multicolumn{2}{c}{ Chieffi \& Limongi (2004) } & Woosley \& Weaver (1995) \\
\hline \multirow{3}{*}{ Salpeter } & top-heavy & Salpeter & top-heavy & Salpeter & top-heavy \\
$\mathrm{Si}$ & 0.113 & 0.124 & 0.163 & 0.183 & 0.127 & 0.132 \\
$\mathrm{Fe}$ & 0.090 & 0.091 & 0.132 & 0.135 & 0.109 & 0.106 \\
$\mathrm{Ni}$ & 0.005 & 0.005 & 0.014 & 0.013 & 0.014 & 0.013 \\
\hline
\end{tabular}

Table A.2. Yields of Si and Ni relative to Fe in solar units of Anders \& Grevesse (1989) for the same SNe models used in Table A.1.

\begin{tabular}{lccccccc}
\hline \hline & \multicolumn{7}{c}{ SNIa } \\
\hline Ratio & W7 & W70 & WDD1 & WDD2 & WDD3 & CDD1 & CDD2 \\
\hline $\mathrm{Si} / \mathrm{Fe}$ & 0.55 & 0.48 & 1.07 & 0.69 & 0.48 & 1.13 & 0.63 \\
$\mathrm{Ni} / \mathrm{Fe}$ & 4.20 & 3.23 & 1.42 & 1.85 & 2.09 & 1.35 & 1.85 \\
\hline
\end{tabular}

\begin{tabular}{ccccccc}
\hline \hline & \multicolumn{2}{c}{ SNcc } \\
\hline Ratio & \multicolumn{2}{c}{ Nomoto etal. (2006) } & \multicolumn{2}{c}{ Chieffi \& Limongi (2004) } & \multicolumn{2}{c}{ Woosley \& Weaver (1995) } \\
\hline & Salpeter & top-heavy & Salpeter & top-heavy & Salpeter & top-heavy \\
$\mathrm{Si} / \mathrm{Fe}$ & 3.28 & 3.58 & 3.22 & 3.56 & 3.07 & 3.26 \\
$\mathrm{Ni} / \mathrm{Fe}$ & 1.43 & 1.38 & 2.71 & 2.43 & 3.24 & 2.99 \\
\hline
\end{tabular}


S. De Grandi and S. Molendi: Metal abundances in the cool cores of galaxy clusters, Online Material $p 3$

Table 1. Starting sample of 32 galaxy clusters extracted from the X-ray flux limited B55 sample (Edge et al. 1990).

\begin{tabular}{|c|c|c|c|c|c|c|c|}
\hline \multirow[t]{2}{*}{ Cluster } & \multirow[t]{2}{*}{ 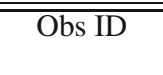 } & \multirow[t]{2}{*}{$\overline{z^{a}}$} & 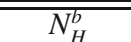 & \multicolumn{3}{|c|}{ Exp.time $^{c}$} & \multirow{2}{*}{$\begin{array}{c}r_{\text {cool }} / 2^{d} \\
\operatorname{arcmin}(\mathrm{kpc})\end{array}$} \\
\hline & & & $10^{22} \mathrm{~cm}^{-2}$ & MOS1 & MOS2 & $p n$ & \\
\hline Abell 85 & 0065140101 & 0.0551 & 0.0342 & 12.5 & 12.5 & 9.7 & $1.137(73)$ \\
\hline Abell 262 & 0109980101 & 0.0163 & 0.0538 & 23.8 & 23.9 & 19.2 & $2.613(52)$ \\
\hline AWM7 & 0135950301 & 0.0172 & 0.0983 & 30.7 & 30.7 & 28.7 & $2.473(52)$ \\
\hline Abell 3112 & 0105660101 & 0.0750 & 0.0261 & 23.3 & 23.3 & 18.6 & $1.124(96)$ \\
\hline Perseus & 0085110101 & 0.0179 & 0.1410 & 53.7 & 53.7 & 51.3 & 4.263 (93) \\
\hline 2 A $0335+096$ & 0147800201 & 0.0349 & 0.1780 & 80.2 & 80.9 & 73.9 & $2.596(108)$ \\
\hline Abell 478 & 0109880101 & 0.0881 & 0.1510 & 28.7 & 79.0 & 42.6 & $1.032(102)$ \\
\hline Abell 496 & 0135120201 & 0.0329 & 0.0458 & 17.8 & 17.6 & 14.1 & $1.397(55)$ \\
\hline PKS $0745-191$ & 0105870101 & 0.1028 & 0.4240 & 18.9 & 18.9 & 9.8 & 0.944 (107) \\
\hline Hydra A & 0109980301 & 0.0549 & 0.0494 & 18.6 & 19.8 & 15.8 & $1.266(81)$ \\
\hline Abell 1060 & 0206230101 & 0.0126 & 0.0490 & 40.0 & 41.5 & 33.2 & $2.588(40)$ \\
\hline Virgo (M87) & 0200920101 & 0.0044 & 0.0254 & 77.8 & 79.4 & 70.0 & $9.352(51)$ \\
\hline Centaurus & 0046340101 & 0.0114 & 0.0806 & 40.7 & 41.3 & 40.3 & $2.928(41)$ \\
\hline Abell 1644 & 0010420201 & 0.0473 & 0.0499 & 15.1 & 15.2 & 12.8 & $0.521(29)$ \\
\hline Abell 1650 & 0093200101 & 0.0838 & 0.0156 & 38.1 & 36.6 & 34.4 & $0.878(83)$ \\
\hline Abell 1651 & 0203020101 & 0.0849 & 0.0181 & 10.2 & 10.8 & 6.9 & 0.669 (64) \\
\hline Abell 1689 & 0093030101 & 0.1830 & 0.0182 & 37.5 & 37.6 & 32.6 & $0.520(96)$ \\
\hline Abell 3558 & 0107260101 & 0.0480 & 0.0389 & 44.1 & 44.0 & 38.9 & $0.602(34)$ \\
\hline Abell 3562 & 0105261301 & 0.0490 & 0.0385 & 40.3 & 40.2 & 38.0 & $0.834(48)$ \\
\hline Abell 3571 & 0086950201 & 0.0391 & 0.0371 & 24.3 & 24.5 & 16.5 & $1.119(52)$ \\
\hline Abell 1795 & 0097820101 & 0.0625 & 0.0119 & 39.5 & 39.6 & 22.7 & $1.232(89)$ \\
\hline Abell 2029 & 0111270201 & 0.0773 & 0.0304 & 10.7 & 11.2 & 10.3 & 1.059 (93) \\
\hline Abell 2052 & 0109920101 & 0.0355 & 0.0272 & 30.3 & 30.3 & 26.5 & 1.747 (74) \\
\hline MKW3s & 0109930101 & 0.0450 & 0.0303 & 38.7 & 38.3 & 34.1 & $1.620(86)$ \\
\hline Abell 2065 & 0202080201 & 0.0726 & 0.0295 & 20.7 & 20.7 & 16.6 & 0.338 (28) \\
\hline Abell 2063 & 0200120401 & 0.0349 & 0.0298 & 8.1 & 8.9 & 5.4 & $1.152(48)$ \\
\hline Abell 2199 & 0008030201 & 0.0302 & 0.0086 & 14.6 & 14.6 & 10.0 & $1.985(72)$ \\
\hline Abell 2204 & 0112230301 & 0.1523 & 0.0567 & 19.9 & 20.2 & 13.7 & 0.629 (100) \\
\hline Cygnus A & 0302800101 & 0.0561 & 0.3490 & 22.2 & 22.2 & 20.2 & $1.041(68)$ \\
\hline Abell 2597 & 0147330101 & 0.0852 & 0.0249 & 52.2 & 53.3 & 48.5 & $0.792(76)$ \\
\hline Abell 4038 & 0204460101 & 0.0300 & 0.0155 & 29.4 & 29.2 & 27.6 & 1.859 (67) \\
\hline Abell $4059^{e}$ & 0109950101 & 0.0475 & 0.0110 & 12.7 & 13.7 & 7.0 & $1.378(77)$ \\
\hline Abell 4059 & 0109950201 & 0.0475 & 0.0110 & 23.3 & 23.2 & 19.7 & $1.378(77)$ \\
\hline
\end{tabular}

All objects are cool core clusters with a mass deposition rate different from zero (Peres et al. 1998).

Notes: ${ }^{a}$ redshifts taken from the NASA Extragalactic Database; ${ }^{b}$ Galactic photoelectric absorption extracted from the HEASARCH Database (LAB survey Kalberla et al. 2005); ${ }^{c}$ net exposure times for the three EPIC instruments after data cleaning in ks; ${ }^{d}$ extraction radius is half the cooling radius from Peres et al. (1998); ${ }^{e}$ the two observations of Abell 4059 were merged before the spectral analysis.

Table 2. Relative differences between metal measurements made with the three EPIC instruments MOS1, MOS2 and pn computed over the whole sample.

\begin{tabular}{lccc}
\hline \hline mekal model & & & \\
\hline Metal $X$ & $\left(X_{\mathrm{MOS} 1}-X_{\mathrm{MOS} 2}\right) / X_{\mathrm{MOS} 2}$ & $\left(X_{\mathrm{MOS} 1}-X_{p n}\right) / X_{p n}$ & $\left(X_{\mathrm{MOS} 2}-X_{p n}\right) / X_{p n}$ \\
\hline Fe & $0.02 \pm 0.01$ & $0.07 \pm 0.01$ & $0.02 \pm 0.02$ \\
$+3 \%$ syst.err. & $0.02 \pm 0.02$ & $0.04 \pm 0.02$ & $0.01 \pm 0.02$ \\
\hline Si & $0.01 \pm 0.02$ & $0.19 \pm 0.03$ & $0.10 \pm 0.03$ \\
$+3 \%$ syst.err. & $-0.03 \pm 0.04$ & $0.11 \pm 0.04$ & $0.08 \pm 0.04$ \\
\hline Ni & $-0.12 \pm 0.08$ & $0.14 \pm 0.11$ & $0.01 \pm 0.09$ \\
$+3 \%$ syst.err. & $-0.13 \pm 0.08$ & $0.12 \pm 0.11$ & $0.00 \pm 0.10$ \\
\hline
\end{tabular}

\begin{tabular}{lccc}
\hline \hline apec model & & & \\
\hline Metal $X$ & $\left(X_{\mathrm{MOS} 1}-X_{\mathrm{MOS} 2}\right) / X_{\mathrm{MOS} 2}$ & $\left(X_{\mathrm{MOS} 1}-X_{p n}\right) / X_{p n}$ & $\left(X_{\mathrm{MOS} 2}-X_{p n}\right) / X_{p n}$ \\
\hline $\mathrm{Fe}$ & $0.03 \pm 0.01$ & $0.07 \pm 0.01$ & $0.02 \pm 0.02$ \\
$+3 \%$ syst.err. & $0.02 \pm 0.02$ & $0.04 \pm 0.02$ & $0.01 \pm 0.02$ \\
\hline $\mathrm{Si}$ & $0.01 \pm 0.03$ & $0.22 \pm 0.03$ & $0.13 \pm 0.04$ \\
$+3 \%$ syst.err. & $-0.03 \pm 0.05$ & $0.13 \pm 0.04$ & $0.11 \pm 0.04$ \\
\hline $\mathrm{Ni}$ & $-0.16 \pm 0.09$ & $0.13 \pm 0.14$ & $0.01 \pm 0.11$ \\
$+3 \%$ syst.err. & $-0.17 \pm 0.10$ & $0.12 \pm 0.14$ & $0.01 \pm 0.11$ \\
\hline
\end{tabular}

For each element in the first line only statistical errors are accounted for, in the second line a $3 \%$ systematic error is added in quadrature to each abundance measurement (see discussion in Sect. 4.3). 
S. De Grandi and S. Molendi: Metal abundances in the cool cores of galaxy clusters, Online Material p 4

Table 3. Measurements of silicon, iron and nickel abundances relative to the solar value (Anders \& Grevesse 1989) for the final sample (26 clusters for $\mathrm{Si}$ and $\mathrm{Fe}, 23$ for $\mathrm{Ni}$ ) from the 4T mekal spectral model.

\begin{tabular}{rlccc}
\hline \hline Seq. & Cluster & $\mathrm{Si}$ & $\mathrm{Fe}$ & $\mathrm{Ni}$ \\
\hline 1 & Perseus & $0.77 \pm 0.03$ & $0.49 \pm 0.02$ & $1.27 \pm 0.11$ \\
2 & Virgo(M87) & $0.95 \pm 0.07$ & $0.41 \pm 0.02$ & \\
3 & 2A 0335 +096 & $0.73 \pm 0.04$ & $0.48 \pm 0.02$ & $1.03 \pm 0.06$ \\
4 & Centaurus & $1.62 \pm 0.16$ & $0.94 \pm 0.03$ & $2.50 \pm 0.17$ \\
5 & Abell 478 & $0.36 \pm 0.08$ & $0.43 \pm 0.02$ & $0.62 \pm 0.16$ \\
6 & Abell 1795 & $0.68 \pm 0.04$ & $0.47 \pm 0.02$ & $0.89 \pm 0.11$ \\
7 & Abell 2597 & $0.40 \pm 0.09$ & $0.37 \pm 0.02$ & $0.79 \pm 0.51$ \\
8 & Abell 4038 & $0.49 \pm 0.08$ & $0.42 \pm 0.03$ & $1.50 \pm 0.23$ \\
9 & Abell 1060 & $0.73 \pm 0.07$ & $0.42 \pm 0.02$ & \\
10 & MKW3s & $0.78 \pm 0.07$ & $0.48 \pm 0.02$ & $1.36 \pm 0.75$ \\
11 & Abell 2052 & $0.77 \pm 0.03$ & $0.55 \pm 0.02$ & $1.35 \pm 0.09$ \\
12 & Abell 2199 & $0.92 \pm 0.10$ & $0.45 \pm 0.02$ & $1.11 \pm 0.41$ \\
13 & Abell 2029 & $0.61 \pm 0.15$ & $0.58 \pm 0.03$ & $1.66 \pm 0.86$ \\
14 & Abell 3112 & $0.77 \pm 0.10$ & $0.58 \pm 0.04$ & $1.17 \pm 0.43$ \\
15 & HYDRA A & $0.46 \pm 0.11$ & $0.39 \pm 0.02$ & $0.75 \pm 0.13$ \\
16 & Abell 496 & $0.85 \pm 0.06$ & $0.55 \pm 0.02$ & $1.72 \pm 0.37$ \\
17 & AWM7 & $1.13 \pm 0.07$ & $0.64 \pm 0.02$ & $1.24 \pm 0.30$ \\
18 & ABELL 4059 & $0.78 \pm 0.03$ & $0.59 \pm 0.04$ & $1.00 \pm 0.12$ \\
19 & Abell 3571 & $0.52 \pm 0.15$ & $0.48 \pm 0.03$ & $1.81 \pm 0.09$ \\
20 & Abell 1650 & $0.36 \pm 0.16$ & $0.57 \pm 0.03$ & $1.11 \pm 0.62$ \\
21 & Abell 1689 & $0.40 \pm 1.68$ & $0.33 \pm 0.03$ & $1.96 \pm 0.33$ \\
22 & PKS 0745 191 & $0.61 \pm 0.22$ & $0.43 \pm 0.03$ & $0.49 \pm 0.22$ \\
23 & Abell 262 & $0.93 \pm 0.06$ & $0.53 \pm 0.06$ & \\
24 & Abell 2204 & $0.83 \pm 0.24$ & $0.53 \pm 0.03$ & $1.27 \pm 0.49$ \\
25 & Abell 85 & $0.94 \pm 0.23$ & $0.55 \pm 0.02$ & $2.49 \pm 0.74$ \\
26 & Abell 3558 & $0.84 \pm 0.36$ & $0.49 \pm 0.02$ & $1.68 \pm 1.15$ \\
\hline
\end{tabular}

Clusters are sorted by decreasing counts statistics in the pn. Errors on abundances include a 3\% systematic error as detailed in Sect. 4.3 .

Table 4. Averages of $\mathrm{Si}, \mathrm{Fe}, \mathrm{Ni}$ abundances and $\mathrm{Si} / \mathrm{Fe}, \mathrm{Ni} / \mathrm{Fe}$ ratios for the cool core cluster sample in solar units.

\begin{tabular}{lcc}
\hline \hline mekal model & & \\
\hline Metal & mean & scatter \\
\hline Si & $0.75 \pm 0.03$ & $0.22 \pm 0.03$ \\
Si no Cent. & $0.72 \pm 0.03$ & $0.17 \pm 0.02$ \\
\hline Fe & $0.51 \pm 0.02$ & $0.11 \pm 0.01$ \\
Fe no Cent. & $0.49 \pm 0.01$ & $0.07 \pm 0.01$ \\
\hline $\mathrm{Ni}$ & $1.28 \pm 0.08$ & $0.45 \pm 0.06$ \\
$\mathrm{Ni}$ no Cent. & $1.18 \pm 0.07$ & $0.34 \pm 0.06$ \\
\hline $\mathrm{Si} / \mathrm{Fe}$ & $1.47 \pm 0.05$ & $0.27 \pm 0.05$ \\
$\mathrm{Si} / \mathrm{Fe}$ no Cent. & $1.45 \pm 0.05$ & $0.30 \pm 0.05$ \\
\hline $\mathrm{Ni} / \mathrm{Fe}$ & $2.41 \pm 0.13$ & $0.60 \pm 0.12$ \\
$\mathrm{Ni} / \mathrm{Fe}$ no Cent. & $2.40 \pm 0.14$ & $0.63 \pm 0.13$ \\
\hline
\end{tabular}

\begin{tabular}{lcc}
\hline \hline apec model & & \\
\hline Metal & mean & scatter \\
\hline $\mathrm{Si}$ & $0.83 \pm 0.04$ & $0.23 \pm 0.03$ \\
$\mathrm{Si}$ no Cent. & $0.80 \pm 0.03$ & $0.19 \pm 0.03$ \\
\hline $\mathrm{Fe}$ & $0.53 \pm 0.02$ & $0.12 \pm 0.01$ \\
Fe no Cent. & $0.51 \pm 0.01$ & $0.08 \pm 0.01$ \\
\hline $\mathrm{Ni}$ & $1.07 \pm 0.10$ & $0.53 \pm 0.07$ \\
$\mathrm{Ni}$ no Cent. & $0.96 \pm 0.08$ & $0.39 \pm 0.06$ \\
\hline $\mathrm{Si} / \mathrm{Fe}$ & $1.56 \pm 0.05$ & $0.25 \pm 0.05$ \\
$\mathrm{Si} / \mathrm{Fe}$ no Cent. & $1.56 \pm 0.05$ & $0.26 \pm 0.05$ \\
\hline $\mathrm{Ni} / \mathrm{Fe}$ & $1.88 \pm 0.14$ & $0.67 \pm 0.14$ \\
$\mathrm{Ni} / \mathrm{Fe}$ no Cent. & $1.83 \pm 0.15$ & $0.67 \pm 0.15$ \\
\hline
\end{tabular}

Mean values are given both for the total sample and with the exclusion of the peculiar Centaurus cluster. Average abundance values and intrinsic scatters are computed with the maximum likelihood method described in Maccacaro et al. (1988). 
S. De Grandi and S. Molendi: Metal abundances in the cool cores of galaxy clusters, Online Material $p 5$

Table 5. Supernovae type Ia mass fraction, $\xi=M_{\mathrm{Fe}, \mathrm{SNI}} /\left(M_{\mathrm{Fe}, \mathrm{SNIa}}+M_{\mathrm{Fe}, \mathrm{SNcc}}\right)$, obtained for different theoretical SNe silicon-to-iron and nickel-toiron ratios from the observed $\mathrm{Si} / \mathrm{Fe}$ and $\mathrm{Ni} / \mathrm{Fe}$ ratios.

\begin{tabular}{|c|c|c|c|c|c|c|c|}
\hline mekal model & \multicolumn{7}{|c|}{$\overline{\text { SNIa }}$} \\
\hline SNcc & W7 & W70 & WDD1 & WDD2 & WDD3 & CDD1 & CDD2 \\
\hline No06,S & $0.528-0.571$ & $0.515-0.734$ & & & & & \\
\hline No06,T & $0.575-0.579$ & $0.562-0.760$ & & & & & \\
\hline CL04,S & & $0.505-0.582$ & & $0.546-0.789$ & $0.505-0.728$ & & $0.534-0.770$ \\
\hline CL04,T & & $0.559-0.728$ & & $0.600-0.727$ & $0.559-0.758$ & & $0.588-0.727$ \\
\hline W95,S & & & $0.616-0.677$ & $0.518-0.775$ & $0.476-0.712$ & $0.635-0.652$ & $0.505-0.756$ \\
\hline W95,T & & & & $0.554-0.792$ & $0.512-0.732$ & & $0.541-0.774$ \\
\hline apec model & & & & $\overline{\text { SNIa }}$ & & & \\
\hline SNcc & W7 & W70 & $\overline{\text { WDD1 }}$ & WDD2 & WDD3 & CDD1 & CDD2 \\
\hline No06,S & & $0.475-0.511$ & & $0.514-0.764$ & $0.475-0.707$ & & $0.502-0.747$ \\
\hline No06,T & & & & $0.564-0.789$ & $0.526-0.735$ & & $0.553-0.773$ \\
\hline CL04,S & & & $0.591-0.886$ & $0.502-0.759$ & $0.581-0.701$ & $0.608-0.841$ & $0.490-0.741$ \\
\hline CL04,T & & & $0.647-0.855$ & $0.561-0.787$ & $0.523-0.734$ & $0.663-0.799$ & $0.549-0.771$ \\
\hline W95,S & & & $0.560-0.885$ & $0.640-0.744$ & & $0.577-0.885$ & $0.640-0.725$ \\
\hline W95,T & & & $0.598-0.895$ & $0.561-0.763$ & & $0.615-0.868$ & $0.561-0.745$ \\
\hline
\end{tabular}

SNIa yields are computed from the full set of models for deflagration (W7, W70) and delayed detonation (WDDs, CDDs) scenarios by Iwamoto et al. (1999). SNcc yields are integrated over a Salpeter $(x=1.35)$ or a AY top-heavy $(x=0.95)$ IMF, with abundance of the progenitor $Z=0.02$. Models for SNcc are taken from Nomoto et al. (2006, No06), Chieffi \& Limongi (2004, CL04) and Woosley \& Weaver (1995, W95).

Table 6. Supernovae type Ia fraction, $f=\mathrm{SNIa} /(\mathrm{SNIa}+\mathrm{SNcc})$, obtained for different theoretical $\mathrm{SNe}$ models from the observed $\mathrm{Si} / \mathrm{Fe}$ and Ni/Fe abundance ratios. SNIa and SNcc models are the same as in Table 5.

\begin{tabular}{|c|c|c|c|c|c|c|c|}
\hline mekal model & \multicolumn{7}{|c|}{ SNIa } \\
\hline SNcc & W7 & W70 & WDD1 & WDD2 & WDD3 & CDD1 & CDD2 \\
\hline No06,S & $0.117-0.136$ & $0.110-0.244$ & & & & & \\
\hline No06,T & $0.139-0.141$ & $0.132-0.272$ & & & & & \\
\hline CL04,S & & $0.149-0.193$ & & $0.168-0.384$ & $0.134-0.289$ & & $0.154-0.348$ \\
\hline CL04,T & & $0.182-0.320$ & & $0.204-0.313$ & $0.165-0.327$ & & $0.188-0.302$ \\
\hline W95,S & & & $0.207-0.254$ & $0.129-0.322$ & $0.102-0.237$ & $0.226-0.239$ & $0.118-0.289$ \\
\hline W95,T & & & & $0.143-0.338$ & $0.113-0.250$ & & $0.131-0.304$ \\
\hline apec model & & & & $\overline{\text { SNIa }}$ & & & \\
\hline$\overline{S N c c}$ & W7 & $\overline{\mathrm{W} 70}$ & $\overline{\text { WDD1 }}$ & $\overline{\text { WDD2 }}$ & $\overline{\text { WDD3 }}$ & CDD1 & CDD2 \\
\hline No06,S & & $0.096-0.109$ & & $0.107-0.270$ & $0.086-0.200$ & & $0.098-0.243$ \\
\hline No06,T & & & & $0.130-0.301$ & $0.104-0.225$ & & $0.119-0.272$ \\
\hline CL04,S & & & $0.221-0.606$ & $0.144-0.345$ & $0.174-0.262$ & $0.239-0.517$ & $0.133-0.313$ \\
\hline CL04,T & & & $0.269-0.543$ & $0.179-0.388$ & $0.145-0.300$ & $0.290-0.453$ & $0.166-0.354$ \\
\hline W95,S & & & $0.172-0.556$ & $0.197-0.286$ & & $0.186-0.564$ & $0.189-0.258$ \\
\hline W95,T & & & $0.191-0.574$ & $0.147-0.301$ & & $0.207-0.517$ & $0.141-0.272$ \\
\hline
\end{tabular}

